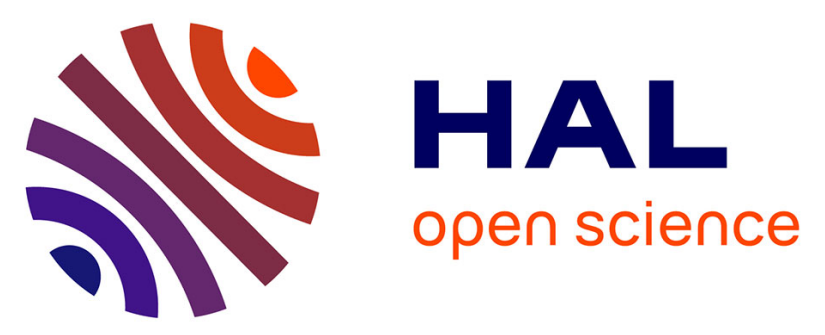

\title{
Spectrally selective pencil-beam navigator for motion compensation of MR-guided high-intensity focused ultrasound therapy of abdominal organs
}

Max O Köhler, Baudouin Denis de Senneville, Bruno Quesson, Chrit T W Moonen, Mario Ries

\section{To cite this version:}

Max O Köhler, Baudouin Denis de Senneville, Bruno Quesson, Chrit T W Moonen, Mario Ries. Spectrally selective pencil-beam navigator for motion compensation of MR-guided high-intensity focused ultrasound therapy of abdominal organs. Magnetic Resonance in Medicine, 2011, 66 (1), pp.102-111. 10.1002/mrm.22784 . hal-01578209

\section{HAL Id: hal-01578209 \\ https://hal.science/hal-01578209}

Submitted on 28 Aug 2017

HAL is a multi-disciplinary open access archive for the deposit and dissemination of scientific research documents, whether they are published or not. The documents may come from teaching and research institutions in France or abroad, or from public or private research centers.
L'archive ouverte pluridisciplinaire HAL, est destinée au dépôt et à la diffusion de documents scientifiques de niveau recherche, publiés ou non, émanant des établissements d'enseignement et de recherche français ou étrangers, des laboratoires publics ou privés. 


\title{
Spectrally selective pencil-beam navigator for motion compensation of MR- guided high-intensity focused ultrasound therapy of abdominal organs
}

\author{
Max O. Köhler ${ }^{1}$, Baudouin Denis de Senneville ${ }^{2}$, Bruno Quesson ${ }^{2}$, Chrit T.W. Moonen ${ }^{2}$, Mario \\ Ries $^{2}$ \\ ${ }^{1}$ Philips Healthcare, Finland, ${ }^{2}$ Laboratory for Molecular and Functional Imaging: From Physiology to \\ Therapy, University of Bordeaux 2, France
}

\author{
Running head: Spectrally selective pencil-beam navigator \\ Max Köhler \\ Address: Äyritie 4, 01510 Vantaa, Finland \\ Tel: +358504151398 \\ Email: max.kohler@philips.com \\ Word count: 5176
}




\begin{abstract}
MR-guided high-intensity focused ultrasound (MR-HIFU) is a noninvasive technique for depositing thermal energy in a controlled manner deep within the body. However, the MR-HIFU treatment of mobile abdominal organs is problematic since motion-related thermometry artifacts need to be corrected and the focal point position must be updated to follow the moving organ in order to avoid damaging healthy tissue. In this paper, a fat-selective pencil-beam navigator is proposed for real-time monitoring and compensation of through-plane motion. As opposed to the conventional spectrally non-selective navigator, the fat-selective navigator does not perturb the water-proton magnetization used for proton resonance frequency shift thermometry. This allows the proposed navigator to be placed directly on the target organ for improved motion estimation accuracy. The spectral and spatial selectivity of the proposed navigator pulse is evaluated through simulations and experiments, and the improved slice tracking performance is demonstrated in vivo by tracking experiments on a human kidney and on a human liver. The direct motion estimation provided by the fat-selective navigator is also shown to enable accurate motion compensated MR-HIFU therapy of in vivo porcine kidney, including motion compensation of thermometry and beam steering based on the observed three-dimensional kidney motion.
\end{abstract}

Keywords: HIFU, MR thermometry, pencil beam, navigator, spectrally selective, beam steering 


\section{Introduction}

High-intensity focused ultrasound (HIFU) is a noninvasive technique for performing local thermal therapy $(1,2)$. The combination of MRI with HIFU (MR-HIFU) for providing image guidance is particularly promising since the excellent soft-tissue contrast of MRI allows for exact target localization and evaluation of treatment outcome $(3,4)$.

The main utility of MRI for HIFU therapy is, however, its ability to produce real-time maps of the instantaneous in vivo temperature distribution in any imaging plane with a high spatial resolution. Temperature images can be obtained by utilizing the linear temperature dependency of the proton resonance frequency (PRF) shift of water and the change in MR phase information between sequentially acquired gradient-echo images $(5,6)$. The relationship between the PRF shift and temperature is nearly independent of tissue type with the exception of adipose tissue whose PRF is almost constant despite variations in temperature $(7,8)$. Consequently, fat suppression or water-selective excitation is required in order to avoid errors in the PRF-based temperature images $(9,10)$.

Several abdominal organs would be prime candidates for MR-HIFU therapy, but are unfortunately subject to rigid or non-rigid motion during the respiratory cycle. This leads to two problems. First, the respiration-induced organ displacement and deformation will modify the local field and thus also the local magnetic field experienced by the target organ. This will result in phase variations and thus temperature artifacts. Second, the focal point of the HIFU-system must also be adaptively repositioned as the organ moves with respect to the external transducer in order to avoid damaging healthy tissue and ensure an efficient heating.

Several approaches for the removal of thermometry artifacts have been proposed in the past, such as multi-baseline corrections to sample periodic changes $(11,12)$ and referenceless phase corrections $(13)$. A recent approach combined multi-baseline corrections with rapid 2D image registration to provide both a removal of thermometry artifacts and a correction of in-plane displacement in real-time (14). Slice tracking based on pencil-beam navigator data can moreover be used to address the remaining throughplane motion $(15,16)$, thus allowing the motion compensation to be extended to three dimensions. This approach was recently shown to enable real-time 3D motion compensated MR-HIFU therapy, including real-time 3D motion compensated MR thermometry and real-time beam steering of the HIFU focal spot according to the obtained 3D motion of the target region (17). 
The conventional pencil-beam navigator is, however, not well-suited for providing the exact motion estimation that is imperative for safe MR-HIFU therapy of mobile organs. For MR-HIFU, the navigator should preferably be placed directly on the target tissue for an exact motion compensation of both thermometry and focal point position. The pencil-beam navigator will thus necessarily intersect the imaging slice or slices that monitor the temperature rise in the target region when used for through-plane motion compensation. Moreover, both the conventional pencil-beam navigator and the imaging slices used for PRF-based thermometry act on the same water-proton magnetization. The navigator will thus perturb the magnetization of the imaging slice and vice versa, which may lead to reduced temperature accuracy, temperature artifacts, and slice tracking errors. The conventional proton-density weighted pencil-beam navigator will also suffer from poor contrast in the abdominal region that may further degrade the tracking performance.

Here, a spectrally selective pencil-beam navigator is presented. A fat-selective pencil-beam navigator should not perturb the water-proton magnetization of the imaging slice, or vice versa, and may thus provide an elegant solution to the problems of the conventional pencil-beam navigator for use in MRHIFU. The proposed RF pulse was evaluated theoretically as well as experimentally. In vivo experiments were performed to assess the slice tracking capabilities of the proposed navigator when placed directly on the kidney and on the liver of a healthy volunteer. Moreover, the efficacy of the fat-selective navigator for providing the accurate through-plane motion estimate necessary for 3D motion compensated MR-HIFU, including compensation of thermometry and focal point position, was evaluated in vivo on the kidney of a pig.

\section{Theory}

Similar to 2D acquisition schemes, such as spiral acquisition, 2D spatial selective excitation can be constructed by coupling an RF waveform with a $2 \mathrm{D}$ gradient waveform that determines the k-space trajectory of the excitation $(18,19)$. Nehrke et al. (16) proposed such an excitation utilizing a spiral kspace trajectory in conjunction with a 1D selective readout for achieving a pencil-beam shaped navigator echo, further improving the approach initially presented by Hardy et al.(20).

Compared to other k-space trajectories, an inward spiral k-space trajectory for 2D RF excitation shows several interesting properties. Most notably, the 2D RF pulse will be inherently self-refocused as the inward spiral trajectory ends in the k-space center (18). The spiral can also be considered to cover the kspace symmetrically, resulting in a linear dependence between the transversal magnetization and the 
applied $B_{1}$ field (19). The spiral trajectory will, moreover, utilize the gradient system very efficiently and thus allows short pulse durations to encode the desired part of $\mathrm{k}$-space.

However, the limitations of the available encoding time and the maximal gradient strength will introduce a circular aperture in k-space within which the radial k-space coverage is discrete. Consequently, the central peak of the spiral excitation point spread function (PSF) has the form of a jinc function surrounded by a series of regularly spaced radial secondary lobes that are interspersed by small amplitude oscillations (21). The first of the evenly spaced radial secondary lobes can be found at

$$
r_{1}=\frac{2 \pi N}{k_{\max }}=\frac{2 \pi N}{3.83} r_{0},
$$

where $N$ is the amount of k-space spiral turns, and $r_{0}$ is the pencil-beam radius that is here defined as the first zero-crossing of the jinc-shaped PSF center peak (16). The amplitude of the oscillations can be attenuated by a suitable apodization function at the expense of a slightly broadened central peak (16). For this purpose, the desired excitation profile is here chosen to be that of a disk with radius equal to the pencil beam. The convolution of this disk-shaped desired excitation profile with the PSF will determine the actual excitation profile, thereby dampening the oscillations between the side lobes of the PSF.

The disadvantages of the conventional pencil-beam excitation for motion compensation of MR-HIFU largely arise because the pencil beam excites the same water-proton magnetization as the imaging slices used for PRF-thermometry. Spectrally selective excitation offers a potentially elegant solution to this problem (10). Water-selective excitation may be used to discard the fat as is necessary for PRF-based thermometry to avoid erroneous temperature readings. A fat-selective pencil beam would therefore avoid perturbing the magnetization in the imaging slice, and vice versa. Furthermore, a spectrally selective pencil beam should provide an improved contrast as compared to a conventional spectrally non-selective pencil beam since most abdominal organs are surrounded by a sheath of fat.

Binomial weighting of the 2D RF pulses can be used to provide the desired spectral selectivity with the prerequisite that the transverse magnetization is refocused between the individual pulses $(22,23)$. The separation in time between the binomial pulses must allow fat and water to reach opposite phase. A phase condition of $1 / 2 \pi$ is usually chosen as this makes the spectral selectivity less sensitive to $B_{0}$ inhomogeneities. However, for the case of binomially weighted 2D RF pulses this is not feasible since the maximum gradient strength and gradient slew rates of most whole-body MR scanners would not allow time for sufficient spatial encoding of the excitation despite the use of spiral k-space trajectories. On a 1.5 
T MR scanner with gradient strength of $21 \mathrm{mT} / \mathrm{m}$ and slew rate of $100 \mathrm{~T} / \mathrm{m} / \mathrm{s}$ as used in this study, the maximum amount of spiral turns would be restricted to 2 if a phase separation of $1 / 2 \pi$ is desired. This would result in an extremely poorly shaped PSF with densely spaced radial side lobes of high amplitude $(16,21)$. The second best alternative is then to opt for a fat-water phase difference of $3 / 2 \pi$. Although the longer fat-water dephasing will degrade the ability of the binomial pulse train to separate water and fat, it does allow for a 12-turn spiral trajectory excitation. This provides a sufficiently dense radial coverage of the k-space to produce a well-defined PSF with a sharp central peak and only a few aliasing rings of small amplitudes situated far from the central peak (16).

Even though individual inward spiral trajectories are inherently self-refocused, gradients need to be applied prior to every $2 \mathrm{D}$ RF pulse following the first pulse of the pulse train in order to obtain the same starting point of the k-space trajectory for each of the 2D RF pulses. The k-space starting point of each spiral trajectory could alternatively be slightly rotated to reduce the peak amplitude of the side lobes (24). This is, however, of limited interest for a train of 12-turn spirals whose first radial side lobe occurs at a distance approximately 20 times the radius of the pencil beam. Furthermore, rotation of the k-space starting point would require a different gradient waveform for each 2D RF pulse, which might make the pulse train more sensitive to eddy currents and $B_{1}$ inhomogeneities $(23,25)$.

Although longer binomial pulse trains provide an improved spectral selectivity with a wider stopband (26), motion compensated MR-HIFU therapy of mobile organs imposes strict real-time constraints. The pulse train used in this paper is therefore limited to three binomially weighted 2D RF pulses as a compromise between the requirements on temporal resolution and spectral selectivity. The sequence diagram of the proposed 2D RF pulse train is shown in Fig. 1. The individual 2D RF pulses of the pulse train use a 12-turn spiral k-space trajectory and consecutive pulses are separated by an interval of $6.91 \mathrm{~ms}$ corresponding to a fat-water phase difference of $3 / 2 \pi$ at $1.5 \mathrm{~T}$. This gives rise to a total duration of 19.9 ms for the 1-2-1 binomially weighted 2D RF pulse train. Pulse trains of 2D RF pulses based on spiral trajectories have previously been proposed for excitation of three dimensional volumes (24) and for compensation of field inhomogeneities (27).

\section{Materials \& Methods}

\section{Simulations}

The theoretical performance of the proposed 1-2-1 binomial pencil-beam excitation was evaluated using Bloch-simulation software (http://www-mrsrl.stanford.edu/ brian/bloch/) ported to IDL 6.1.1 (ITT Corp., 
White Plaines, NY). The diameter of the simulated pencil beam was $30 \mathrm{~mm}$ and the total flip angle of the pulse train was $25^{\circ}$. Relaxation was omitted from the simulations.

\section{MR imaging}

The spectrally selective 1-2-1 binomial pulse train of 2D spiral excitations was implemented on a $1.5 \mathrm{~T}$ Philips Achieva scanner (Philips Healthcare, Best, The Netherlands). The implementation was based on the existing implementation of the spiral encoded pencil-beam navigator that is corrected for nonuniform sampling density and short-term eddy currents (16). The intended diameter of the pencil-beam navigator was $30 \mathrm{~mm}$ and the total flip angle of the pulse train was $25^{\circ}$.

The excitation profile of the binomial spiral excitation pulse train was obtained by using this 2D RF pulse train as an excitation pulse coupled with a turbo spin echo (TSE) readout (TR = $1000 \mathrm{~ms}, \mathrm{TE}=58 \mathrm{~ms}$, FOV $=300 \times 300 \mathrm{~mm}^{2}$, matrix $=128 \times 128$, slice thickness $=7 \mathrm{~mm}$ ), where the refocusing pulse was made slice selective perpendicularly to the pencil beam axis. The target was a homogenous manganesechloride doped phantom.

The slice tracking capabilities of the proposed fat-selective navigator were evaluated in vivo by comparing the positional data obtained with the spectrally selective navigator to that of the non-selective 12-turn spiral navigator when imaging a human kidney and a human liver under free breathing. The experiments were performed on two separate volunteers from whom informed consent was obtained. An axial imaging slice was chosen and the navigator was placed perpendicularly to the imaging slice in the superior-inferior (SI) direction across the fat capsule at the lower apex of the right kidney in the kidney experiment, and across the gallbladder and the surrounding fat in the liver experiment. The positioning of the imaging slice and the navigator for the kidney and the liver experiment is shown in Fig. 2. The sequence used for imaging in both experiments was a single-shot gradient-echo echo planar imaging (EPI) sequence with TR $=110 \mathrm{~ms}, \mathrm{TE}=48 \mathrm{~ms}$, flip angle $=35^{\circ}, \mathrm{FOV}=300 \times 195 \mathrm{~mm}^{2}$, matrix $=128 \times$ 83 , slice thickness $=6 \mathrm{~mm}$, and a 1-2-1 binomial water-selective excitation pulse. One-dimensional navigator profiles were acquired before each dynamic repetition of the imaging sequence by performing a readout along the axis of the excited pencil beam. The imaging slice position was adaptively corrected in real-time based on the estimated target displacement as obtained via correlation analysis of subsequently acquired navigator profiles $(15,28)$. The duration of the single non-selective 2D RF pulse was $6.1 \mathrm{~ms}$, whereas the duration of the fat-selective 2D RF pulse train was $19.9 \mathrm{~ms}$. The duration of the 1D readout 
was in turn $6.6 \mathrm{~ms}$, resulting in a total duration of 12.7 and $26.5 \mathrm{~ms}$ for the non-selective and fat-selective navigators, respectively.

\section{MR-HIFU platform}

The MR-HIFU experiment was performed on a Sonalleve MR-HIFU platform (Philips Healthcare, Vantaa, Finland) that was integrated into the 1.5 T Philips Achieva MR scanner. The MR-HIFU platform consisted of a HIFU transducer embedded in a water-filled MR table top, an RF generator, and a workstation dedicated to therapy control. The 256 element HIFU transducer (Imasonic, Voray sur l'Ognon, France) had spherical shell geometry with a focal length of $12 \mathrm{~cm}$ and aperture of $13 \mathrm{~cm}$, and was operated at 1.2 MHz. The measured focal point size was $1 \times 1 \times 7 \mathrm{~mm}^{3}$. More details on the MRHIFU platform can be found in Ref. (29).

\section{MR thermometry}

The temperature rise induced by the HIFU ablation of the in vivo porcine kidney was monitored in a single axial slice with the proposed fat-selective navigator placed perpendicularly to the imaging slice across the fat capsule of the kidney. The dynamic images used for MR thermometry were acquired with a single-shot gradient-echo EPI sequence with the following acquisition parameters: $\mathrm{TR}=100 \mathrm{~ms}, \mathrm{TE}=48$ $\mathrm{ms}$, flip angle $=35^{\circ}, \mathrm{FOV}=300 \times 195 \mathrm{~mm}^{2}$, matrix $=128 \times 83$, and slice thickness $=6 \mathrm{~mm}$. A 1-2-1 binomial water-selective RF pulse was used to suppress the signal of the fat and avoid thermometry errors. The imaging slice position was adaptively corrected in real-time by modifying the excitation frequency of the water-selective RF pulse according to the estimated kidney motion obtained via correlation analysis of the navigator profiles $(15,28)$.

The acquired images were in-plane realigned prior to the calculation of temperature maps as described in Ref. (14). The principle direction of kidney respiratory motion is the SI direction, which was addressed by slice tracking using the fat-selective navigator. The combination of the estimated $2 \mathrm{D}$ in-plane motion with the navigator-based estimate of the through-plane motion provided the 3D motion estimate necessary for fixing the focal point within the moving kidney as described in Ref. (17). The remaining periodic phase fluctuations due to respiratory motion were corrected using a multi-baseline approach where the look-uptable was indexed according to the kidney displacement estimate obtained by the navigator as explained by Ries et al. (17). The calculation of the PRF-based temperature maps and the employed corrections are described in detail by Roujol et al. (14). 
In vivo three-dimensional motion compensated MR-HIFU

The efficacy of the fat-selective navigator for accurately estimating the through-plane motion required for 3D motion compensated MR-HIFU was evaluated on in vivo porcine kidney. The animal (45 $\mathrm{kg}) \mathrm{was}$ initially anesthetized with a premedication of $10 \mathrm{ml}$ of propofol. General anesthesia was induced and maintained during the experiment with a continuous intravenous infusion of propofol at $1 \mathrm{ml} / \mathrm{min}$. The animal was intubated and ventilated (45 to $100 \%$ O2, respirator paraPac, ResMed SA, France) with a respiratory cycle of approximately $3 \mathrm{~s}$ during the entire procedure. The animal study was performed with the approval of the local ethics committee.

The animal was positioned in a decubitus position on the MR-HIFU table top with the targeted kidney centered on the acoustic window. Following the positioning of the animal, HIFU ablation of the kidney was performed in a single focal point that was steered in real-time according to the estimated 3D motion of the targeted kidney (17). The asymmetric transducer design allowed the electronically steered focal spot to follow the $8 \mathrm{~mm}$ peak-to-peak respiratory motion of the porcine kidney without generating significant secondary lobes. The update of the focal point position was performed synchronously to MRimaging at a frequency of $10 \mathrm{~Hz}$. The ablation was performed using a constant power of $100 \mathrm{~W}$ and sonication duration of $30 \mathrm{~s}$.

An in-house developed real-time reconstructor was used to reduce the time required for MR image reconstruction. The time needed for acquisition (after passage of the k-space center) and data transport (64 ms), image reconstruction and motion estimation (40 ms), and HIFU focal point update (10 ms) resulted in a focal point position update latency of $114 \mathrm{~ms}$. Details on the employed beam steering strategy, including the required calculations and their timings, are given by Ries et al. (17).

\section{Results}

\section{Simulation}

The spectral selectivity of the proposed navigator was demonstrated through simulations at different frequency offsets, where the resulting signal was taken as the complex sum of the transverse magnetization over the 15 -mm radius disk intended for excitation. The results are shown in Fig. 3 for both the fat- and water-selective pencil-beam excitations. For comparison, the spectral response of the conventional non-selective pencil-beam excitation at the resonance frequency of water and fat is also included. Note that the peak-to-valley separation in the spectral response of the binomial pencil-beam 
excitation was $72 \mathrm{~Hz}$, corresponding to one third of the $217 \mathrm{~Hz}$ fat-water resonance frequency shift at 1.5 T. This is a direct consequence of using a fat-water phase difference of $3 / 2 \pi$ instead of the $1 / 2 \pi$ commonly used.

Even though there were additional spectral response peaks outside the desired on-resonance response, the spatial selectivity of the binomially weighted 2D RF pulse train rapidly decreased with increasing frequency offset. This can be seen in Fig. 4 where the excitation profiles at on-resonance and at the first two off-resonance spectral peaks are shown. However, when placing the navigator directly on the abdominal target organ, frequency offsets of approximately $1 \mathrm{ppm}(64 \mathrm{~Hz}$ at $1.5 \mathrm{~T})$ can be expected, i.e. far less than the $2.3 \mathrm{ppm}(145 \mathrm{~Hz})$ frequency offset of the first spectral side peak. For such frequency offsets of around $1 \mathrm{ppm}$, the central peak of the excitation profile had a full width at half maximum (FWHM) within $6 \%$ of the $22.9 \mathrm{~mm}$ on-resonance FWHM. Further off-resonance, the spatial selectivity quickly degenerated to a FWHM of $73 \mathrm{~mm}$ at $3 \mathrm{ppm}$. At even larger frequency offsets, the increased blurring of the central peak caused the magnetization maximum to be located away from the pencil beam axis as can be seen to occur for the excitation profile at the second off-resonance spectral peak in Fig. 4.

The excitation profile central peak of the binomially weighted 2D RF pulse train coincided with that of the conventional spectrally non-selective single $2 \mathrm{D}$ RF pulse at the frequency offsets corresponding to the spectral maxima seen in Fig. 3. Outside these spectral maxima, the excitation profiles of the spectralselective and non-selective pencil-beam excitations did differ. Moreover, according to Eq. 1 the first ring lobes are to be expected at $295 \mathrm{~mm}$ off-axis for a conventional 12-turn spiral encoded 2D RF pulse with an intended pencil beam diameter of $30 \mathrm{~mm}$. Figure 4 shows that this is also the case when the spiral encoded 2D RF pulse is applied in a binomial pulse train at on-resonance.

\section{On-resonance excitation profile comparison}

The central peak of the simulated and experimental excitation profiles are compared in Fig. 5 for the proposed 1-2-1 binomial 2D RF pulse train. Within the limits imposed by the SNR and the imperfect $B_{0}$ shim, the measured excitation profile central peak matched the predicted simulated result of a jinc function with a FWHM of $22.1 \mathrm{~mm}$.

The small oscillations surrounding the central peak deviated somewhat from the ideal radial form in the experimental data by showing slightly larger amplitude in the negative readout direction than in the phase-encoding and positive readout direction. This effect can be attributed to small deviations from the ideal spiral trajectory due to minor gradient system imperfections. 
In vivo imaging

Figure 6a shows a comparison of a slice tracking experiment on a human kidney using a spectrally nonselective conventional pencil-beam navigator and the proposed fat-selective pencil-beam navigator. Figure $6 \mathrm{~b}$ shows a similar comparison of slice tracking experiments using the two navigators on a human liver. In both the kidney and the liver experiment, the spectrally selective navigator showed a good contrast-to-noise ratio and was successful in tracking the organ position over the entire scan duration (1500 images, 3 min scanning, frame-rate 9 images/s) without apparent tracking errors. Although the conventional navigator did have higher SNR, the lower contrast-to-noise ratio caused problems for the correlation algorithm used to determine the target displacement. Moreover, the onset of water-selective MR imaging perturbed the water-magnetization within the non-selective pencil beam in both the kidney and the liver experiment, thus altering the acquired navigator profile (indicated by arrow in Fig. 6). Since the resulting partial saturation was the dominant feature within the non-selective navigator profile in the kidney experiment (Fig. 6a), the navigator-based motion estimation did not follow the kidney motion at all but instead tracked the position of the previously excited imaging plane. As a consequence, the kidney motion was consistently underestimated as can seen by comparing the amplitude of the displacement estimate with the motion of the darker grey band in the middle of the navigator profile. The partial saturation caused by the imaging slice was less dominant in the non-selective navigator profile of the liver experiment (Fig. 6b) due to the higher contrast. Consequently, the motion estimation based on the nonselective navigator was successful during most of the liver experiment. However, it failed to follow the motion of the liver through the entire scan, particularly towards the end of the scan where the motion estimate was sporadic. This can be appreciated by noting that the red displacement estimate does not match the smooth motion of the navigator profile features (e.g. the motion of the fat that appears dark at the bottom of the profile).

In theory, there should be no alteration of the navigator profile at onset of the MR imaging for the fatselective navigator, although in practice a very slight alteration could be seen in both the kidney and liver experiments that can be attributed to off-resonance effects. The magnitude of the alteration was, however, insignificant and did not have any effect on the tracking performance in either experiment. The barely visible alteration of the navigator profile at onset of imaging indicates that the water-selective MR imaging does not notably affect the lipid-proton magnetization excited by the fat-selective navigator. The lower SNR of the fat-selective navigator did, however, result in a slight jitter in the motion estimate.

\section{In vivo motion compensated MR-HIFU}


Figure 7 shows the 3D motion compensated temperature map obtained at the end of a $30 \mathrm{~s}$ beam-steered HIFU sonication on in vivo porcine kidney. The temporal evolution of the temperature at the target point within the kidney can in turn be seen in Fig. 8 for both the beam steered sonication and for a stationary HIFU sonication. Figure 8 also shows the kidney displacement in the SI direction as obtained from the proposed fat-selective navigator that was used for both slice tracking and beam steering. The peak temperature for the stationary HIFU sonication experiment was found to be $6.0{ }^{\circ} \mathrm{C}$ and thus $25 \%$ lower than the peak value of $8.1^{\circ} \mathrm{C}$ for the beam steered experiment. The fat-selective navigator enabled the slice and the HIFU-beam to remain locked on the target area for the entire duration of the experiment (5 $\min$ ). No interference between the fat-selective navigator beam and the water-selective imaging slice was observed.

A similar 3D motion compensated experiment was attempted with the non-selective navigator, but in this case the navigator failed to reliably represent the motion pattern of the kidney. Since the correct measurement of the organ displacement is indispensable for multi-baseline thermometry correction, this rendered thermometry impossible and no HIFU ablation was attempted.

\section{Discussion}

In this study, a spectrally selective pencil-beam navigator pulse was designed for direct motion tracking of mobile abdominal organs that are surrounded by islets of fat, such as the kidney or the liver. For such organs, a fat-selective navigator can provide positional information with high contrast-to-noise without interfering with water-selective MR imaging and vice versa. This enables the proposed navigator to be placed directly on the organ of interest for improved accuracy in the monitoring of the organ motion, instead of indirectly estimating the organ motion based on the observed movement of the diaphragm as is typically done with conventional navigators.

However, spiral encoded 2D spatial selective excitation and frequency selective binomial excitation are both limited by their off-resonance sensitivity when used separately. Hence, a careful analysis of the limitations of the combination of the two methods has to be carried out.

\section{Off-resonance sensitivity of spectral response}

The spectral response of the proposed binomial 2D RF pulse train is by design similar to the original 1-21 binomial 1D RF pulse train $(22,23)$ as seen in Fig. 3. However, the original $1 / 2 \pi$ fat-water phase condition was relaxed to $3 / 2 \pi$ to allow for a sufficiently dense coverage of excitation k-space to create a 
well-defined PSF with only a few sparse low-amplitude aliasing rings and a sharp central peak. This relaxation of the phase condition caused additional spectral response zeroes to occur at one third of the fat-water frequency shift above the frequency of each spectral peak as seen in Fig. 3. Consequently, the width of spectral peaks and valleys is decreased, thereby making the spectral selectivity of the proposed 1-2-1 binomial 2D RF pulse train more sensitive to field inhomogeneities than the original 1-2-1 binomial pulse train. For the conventional binomial 1D RF pulse train (data not shown), a deviation of $0.69 \mathrm{ppm}$ from on-resonance can be tolerated while still keeping the signal of the undesired spectral component (e.g. water) below $10 \%$ of maximum. By comparison, an off-resonance shift of $0.34 \mathrm{ppm}$ will provide a similar signal level from the undesired frequency band for the binomial 2D RF pulse train. The field homogeneity should thus be approximately twice as good for the binomial 1-2-1 2D RF pulse train as for the conventional 1-2-1 binomial pulse in order to give a comparable suppression of the undesired spectral component, with the exact ratio depending on the required level of suppression.

The main contributing factor to the increased off-resonance sensitivity of the binomial 2D RF pulse train spectral response is thus the relaxed phase condition. The spectral selectivity is also, although to a far lesser extent, affected by the off-resonance blurring of the 2D RF pulse spatial profile that alters the offresonance spectral peaks and indirectly affects the bandwidth of the spectral stopband.

\section{Off-resonance sensitivity of spatial response}

The on-resonance spatial response of the proposed 2D RF pulse train is not affected by the binomial weighting of the pulse train. Consequently, the on-resonance excitation profile had a sharp central peak with a FWHM of $22.9 \mathrm{~mm}$ and sparse radial side lobes of low amplitude as seen in Fig. 4 and 5. The first radial side lobe was situated at approximately $30 \mathrm{~cm}$ from the beam axis, thus having only a minor effect on the obtained navigator profile.

At off-resonance frequencies, the evaluation of the spatial selectivity is complicated by that the fact that the PSF of a 2D spatially selective excitation pulse is in general frequency dependent and that this spatial response is furthermore weighted by the binomial pulse train. However, for the proposed RF pulse, the binomial pulse train merely provides a spectral weighting of the spectral-spatial response of the individual 2D RF pulses. Consequently, the spectral response of the binomial 2D RF pulse train is modulated by the spectral response of the individual 2D RF pulses, and the two spectral response curves therefore coincide at the spectral maxima of the binomial 2D RF pulse as seen in Fig. 3. At the corresponding frequency offsets, the blurred off-resonance excitation profile (see Fig. 4) proved to be identical for the single 2D RF pulse and for the binomial pulse train of $2 \mathrm{D}$ RF pulses. 
The spatial response of the single 12-turn spiral excitation pulse and the binomial pulse train of 12-turn spiral excitations did, however, differ outside these spectral maxima due to the binomial weighting. At 1 ppm off-resonance, the FWHM of the excitation profile central peak was $24.0 \mathrm{~mm}$ for the single 2D RF pulse, whereas it was further degraded to $24.2 \mathrm{~mm}$ for the binomial 2D RF pulse train. The influence of the binomial weighting on the excitation profile of the 2D RF pulse train was less significant further offresonance and the spatial selectivity of both the single 2D RF pulse and binomial 2D RF pulse train dropped rapidly. At the first spectral side peak of 2.7 ppm (145 Hz) the FWHM was broadened by 96\%, whereas at the second spectral side peak of $4.5 \mathrm{ppm}(290 \mathrm{~Hz})$ the maximum of the spatial response was no longer located in the desired beam area but shifted outwards toward an aliasing ring.

The binomial weighting only causes a slight further degradation of the spatial profile as compared to the single spiral excitation 2D RF pulse. Severely off-resonant tissue will be poorly localized for both the conventional and the spectrally selective pencil-beam excitation, and any resulting signal will enter the background noise of the navigator profile.

\section{Sensitivity to gradient and $B_{1}$ imperfections}

The high slew rates used by the spiral k-space encoding make the 2D RF pulses particularly sensitive to eddy currents (30). These eddy currents may distort the actual k-space trajectory from its nominal path, thereby causing the RF energy deposition to be misplaced within the excitation k-space. This may in turn degrade the excitation profile. However, no such degradation could be noticed between the theoretical and experimental excitation profile of the spiral 2D RF pulse train in Fig. 5, indicating that the precompensation of the gradient waveforms was successful in correcting for the k-space distortion caused by the eddy currents (16).

Even if no compensation of the eddy currents would have been performed, the identical k-space trajectories and gradient waveforms of the individual 2D RF pulses will induce similar eddy currents for each pulse in the pulse train (25). This should in turn render the spectral selectivity of the proposed binomial 2D RF pulse train insensitive to eddy currents. The symmetrical and identical coverage of excitation k-space by the individual 2D RF pulses in the binomial pulse train also ensures that the spectral response of the proposed pulse is insensitive to $B_{1}$ inhomogeneities (23).

\section{Motion estimation and compensation}


The main disadvantage of the proposed navigator is the time needed for excitation as compared to the original 2D navigator pulse of Nehrke et al. (16). A single 2D RF pulse only required $6.1 \mathrm{~ms}$ for encoding the excitation k-space with a 12-turn spiral, whereas applying this same spiral excitation in a 1-2-1 binomially weighted succession including the appropriate delays required 19.9 ms. Only minor motion is nevertheless likely to occur during the $26.5 \mathrm{~ms}$ required for acquiring the fat-selective navigator echo. Even if assuming a sinusoidal target organ motion with a short breathing cycle of $4 \mathrm{~s}$ and a large motion amplitude of $25 \mathrm{~mm}$ as might occur in the upper abdomen, the organ motion will still only be $0.52 \mathrm{~mm}$ during the fat-selective navigator acquisition. This can be compared to an organ motion of $0.25 \mathrm{~mm}$ during the non-selective navigator acquisition $(12.7 \mathrm{~ms})$. The effect of the target motion on the motion estimate and the slice tracking performance should thus remain negligible for the spectral selective navigator.

The proposed navigator showed an excellent real-time tracking performance of respiration-induced abdominal motion for frame-rates up to 9 images per second as seen in Fig. 6 . The navigator profile of the fat-selective navigator displayed an improved contrast within the abdominal region as compared to the conventional navigator. Even though there should in theory be no interference between the fat-selective navigator and water-proton MR imaging, slight interferences due to off-resonance can occur in practice as seen in Fig. 6. The effect of the interference on the fat-selective navigator profile was, however, barely noticeable and thus had no influence on the tracking algorithm. This is in stark contrast to the conventional non-selective navigator where the partial saturation induced by the MR imaging was a dominating feature within the navigator profile causing severe problems for the motion estimation algorithm. These properties enabled the fat-selective navigator to be placed directly on the abdominal target organ for accurate monitoring of the organ motion without interfering with water-proton MR imaging. For large non-rigid organs such as the liver, the navigator should moreover be placed in the vicinity of the target volume in order for the motion registered by the navigator to correspond to that of the target.

The utility of the proposed navigator for direct and accurate monitoring of the target organ motion was shown with a three-dimensionally motion compensated MR-HIFU sonication on in vivo porcine kidney, where both thermometry and sonication were compensated based on the observed motion. For the motion compensation of MR thermometry, the fat-selective navigator was used for slice tracking as well as providing the index for the look-up-table based multi-baseline compensation. The navigator did not perturb the water-proton magnetization used for PRF-thermometry despite intersecting the imaging slice at the targeted kidney. When only thermometry was motion compensated and no beam steering was performed, the peak target temperature rise was $25 \%$ less than when beam steering was utilized. The 
somewhat small effect can be largely attributed to the mechanical ventilation, which caused the kidney to remain in place for most of the respiratory cycle as seen in Fig. 8. Moreover, the unnaturally rapid kidney motion at exhalation and inhalation enhanced the influence of the focal point update latency. Consequently, the effect of beam steering is likely to be substantially larger during free breathing than mechanical ventilation due to the smooth and continuous organ motion.

The experimental results focused on the fat-selective version of the navigator as it allows for the positioning of the navigator in the field of view of the MR imaging sequence without disturbing the water-proton magnetization, something that the spectrally non-selective and water-selective pencil-beam navigator do not. Moreover, the partial saturation of the non-selective navigator profile caused by the imaging slice may even cause the navigator to track the previously excited imaging plane instead of the organ motion, particularly if it is the dominating feature within the navigator profile as was seen in Fig. 6. A potential disadvantage of the proposed fat-selective navigator arises for lean patients with little fat around their abdominal organs. In such cases, however, a preceding fat-selective image may generally provide a suitable islet of fatty tissue in the vicinity of the organ to place the navigator beam on.

\section{Conclusions}

A spectrally selective navigator was presented for compensation of abdominal organ motion. Robust spectral and 2D spatial selectivity was achieved by combining three pencil-beam excitations into a 1-2-1 binomial pulse train. The resulting spectral selectivity allowed the fat-selective version of the navigator to be placed within the field of view of the MR sequence without interfering with water-proton based images. Direct tracking of the moving organ was achieved with excellent tracking performance due to the reduced interference of the water-proton imaging and the improved contrast-to-noise ratio of the navigator profile. The low interference of the fat-selective navigator on the water-signal, and vice versa, makes it ideal for direct and accurate tracking of organs as well as for PRF based thermometry experiments on mobile organs, where the fat-signal is typically discarded. The fat-selective navigator was also shown to enable accurate real-time motion compensation of MR-HIFU in vivo, where thermometry was compensated for three-dimensional motion and the focal point steered in real-time according to the observed motion.

\section{Acknowledgements}

The authors would like to thank Erkki Vahala of Philips Healthcare for helpful ideas and support on software development. 
References

1. Fry FJ. Precision high intensity focusing ultrasonic machines for surgery. Am J Phys Med 1958;37(3):152-156.

2. Tempany CM, Stewart EA, McDannold N, Quade BJ, Jolesz FA, Hynynen K. MR imaging-guided focused ultrasound surgery of uterine leiomyomas: a feasibility study. Radiology 2003;226(3):897905.

3. Cline HE, Schenck JF, Hynynen K, Watkins RD, Souza SP, Jolesz FA. MR-guided focused ultrasound surgery. J Comput Assist Tomogr 1992;16(6):956-965.

4. Hynynen K, Freund WR, Cline HE, Chung AH, Watkins RD, Vetro JP, Jolesz FA. A clinical, noninvasive, MR imaging-monitored ultrasound surgery method. Radiographics 1996;16(1):185195.

5. Hindman JC. Proton resonance shift of water in gas and liquid states. J Chem Phys 1966;44:45824592.

6. Ishihara Y, Calderon A, Watanabe H, Okamoto K, Suzuki Y, Kuroda K, Suzuki Y. A precise and fast temperature mapping using water proton chemical shift. Magn Reson Med 1995;34(6):814-823.

7. De Poorter J. Noninvasive MRI thermometry with the proton resonance frequency method: study of susceptibility effects. Magn Reson Med 1995;34(3):359-367.

8. Peters RD, Hinks RS, Henkelman RM. Ex vivo tissue-type independence in proton-resonance frequency shift MR thermometry. Magn Reson Med 1998;40(3):454-459.

9. de Zwart JA, Vimeux FC, Delalande C, Canioni P, Moonen CT. Fast lipid-suppressed MR temperature mapping with echo-shifted gradient-echo imaging and spectral-spatial excitation. Magn Reson Med 1999;42(1):53-59.

10. Meyer CH, Pauly JM, Macovski A, Nishimura DG. Simultaneous spatial and spectral selective excitation. Magn Reson Med 1990;15(2):287-304.

11. Denis de Senneville B, Mougenot C, Moonen CT. Real-time adaptive methods for treatment of mobile organs by MRI-controlled high-intensity focused ultrasound. Magn Reson Med 2007;57(2):319-330.

12. Vigen KK, Daniel BL, Pauly JM, Butts K. Triggered, navigated, multi-baseline method for proton resonance frequency temperature mapping with respiratory motion. Magn Reson Med 2003;50(5):1003-1010.

13. Rieke V, Vigen KK, Sommer G, Daniel BL, Pauly JM, Butts K. Referenceless PRF shift thermometry. Magn Reson Med 2004;51(6):1223-1231.

14. Roujol S, Ries M, Quesson B, Moonen C, Denis de SB. Real-time MR-thermometry and dosimetry for interventional guidance on abdominal organs. Magn Reson Med 2010;63(4):1080-1087. 
15. McConnell MV, Khasgiwala VC, Savord BJ, Chen MH, Chuang ML, Edelman RR, Manning WJ. Prospective adaptive navigator correction for breath-hold MR coronary angiography. Magn Reson Med 1997;37(1):148-152.

16. Nehrke K, Börnert P, Groen J, Smink J, Böck JC. On the performance and accuracy of 2D navigator pulses. Magn Reson Imaging 1999;17(8):1173-1181.

17. Ries M, Denis de Senneville B, Roujol S, Berber Y, Quesson B, Moonen C. Real-time 3D target tracking in MRI guided focused ultrasound ablation in moving tissues. Magn Reson Med 2010;in press.

18. Pauly J, Nishimura D, Macovski A. A k-space analysis of small-tip-angle excitation. J Magn Reson 1989;81:43-56.

19. Pauly J, Nishimura D, Macovski A. A linear class of large tip-angle selective excitation pulses. J Magn Reson 1989;82:571-587.

20. Hardy CJ, Pearlman JD, Moore JR, Roemer PB, Cline HE. Rapid NMR cardiography with a halfecho M-mode method. J Comput Assist Tomogr 1991;15(5):868-874.

21. Lauzon ML, Rutt BK. Effects of polar sampling in k-space. Magn Reson Med 1996;36(6):940-949.

22. Hore PJ. Solvent suppression in Fourier transform nuclear magnetic resonance. J Magn Reson 1983;55(2):283-301.

23. Schick F, Forster J, Machann J, Huppert P, Claussen CD. Highly selective water and fat imaging applying multislice sequences without sensitivity to B1 field inhomogeneities. Magn Reson Med 1997;38(2):269-274.

24. Pauly JM, Hu BS, Wang SJ, Nishimura DG, Macovski A. A three-dimensional spin-echo or inversion pulse. Magn Reson Med 1993;29(1):2-6.

25. Block W, Pauly J, Kerr A, Nishimura D. Consistent fat suppression with compensated spectralspatial pulses. Magn Reson Med 1997;38(2):198-206.

26. Schick F. Numerically optimized hard pulse sequences for fast frequency selective excitation and inversion. MAGMA 1993;1(3):158-168.

27. Morrell G, Macovski A. Three-dimensional spectral-spatial excitation. Magn Reson Med 1997;37(3):378-386.

28. Wang Y, Grimm RC, Felmlee JP, Riederer SJ, Ehman RL. Algorithms for extracting motion information from navigator echoes. Magn Reson Med 1996;36(1):117-123.

29. Köhler MO, Mougenot C, Quesson B, Enholm J, Le Bail B, Laurent C, Moonen CT, Ehnholm GJ. Volumetric HIFU ablation under 3D guidance of rapid MRI thermometry. Med Phys 2009;36(8):3521-3535.

30. Takahashi A, Peters T. Compensation of multi-dimensional selective excitation pulses using measured k-space trajectories. Magn Reson Med 1995;34(3):446-456. 
FIG. 1. Gradient and RF waveforms of the proposed 1-2-1 binomial 2D RF pulse train. The excitation kspace encoding is over 12 spiral turns, with a fat-water dephasing corresponding to $3 / 2 \pi$. The RF waveform is weighted according to the nonuniform sampling density.

FIG. 2. Position of navigator (black rectangle) and imaging slice (white rectangle) for the (a) kidney and (b) liver in vivo slice tracking experiments. The navigator pencil beam was placed across the lower apex of the right kidney in the kidney experiment. For the liver experiment, the navigator was placed across the gallbladder and the surrounding fat at the bottom of the liver. The navigators intersected the axial imaging slice perpendicularly in both experiments.

FIG. 3. Spectral response of the water-selective (dashed) and fat-selective (solid) pencil-beam navigator. The two envelopes (dotted) correspond to the non-selective navigator when excited at the fat and water resonance frequency. The distance between neighboring spectral peaks and valleys of $72 \mathrm{~Hz}$ is also shown.

FIG. 4. Excitation profile of the spectrally selective pencil-beam excitation at frequency offsets of $0 \mathrm{~Hz}$ (solid), $145 \mathrm{~Hz}$ (dotted) and $290 \mathrm{~Hz}$ (dashed). The excitation profiles of both the conventional nonselective and the spectral-selective pencil-beam excitation coincide at the frequency offsets corresponding to the spectral peaks of Fig. 3. The full width at half maximum (FWHM) of the excitation profiles at 0 and $145 \mathrm{~Hz}$ are also indicated.

FIG. 5. Comparison of the (a) theoretical and (b) experimental central peak of the on-resonance excitation profile as viewed perpendicularly to the pencil-beam direction. The excitation profile shows a good spatial localization with the central peak in the expected form of a jinc function.

FIG. 6. Comparison of the slice tracking performance of the conventional spectrally non-selective navigator (NS) and the fat-selective navigator (FS) when placed directly on (a) the kidney and (b) the liver. The navigator profiles are plotted vertically with subsequently acquired profiles stacked after each other and the dynamic scan number increasing towards the right. The displacements estimated based on the correlation of the navigator profiles are depicted in red. The navigator profile obtained by the conventional non-selective navigator is altered significantly by the onset of the water-selective MR imaging (indicated by arrow). This effect is much less pronounced for the fat-selective navigator profile. Note that the displacement estimates were shifted relative to the navigator profiles in order not to interfere with the essential features of the navigator profiles. 
FIG. 7. Temperature map of the kidney at the end of a beam-steered HIFU sonication. The motion compensated temperature map is overlaid in color on the axial magnitude image. The transducer and the semi-transparent beam-path overlay as well as the location of the gel pad are shown to clarify the experimental setup. A close-up of the temperature map within the beam path is also shown (top) with the black arrowhead indicating the position of the focus within the kidney. Two additional hotspots (white arrowheads) due near-field heating can also be seen. The proposed fat-selective navigator was used to provide the necessary through-plane motion estimate.

FIG. 8. Temperature rise in the target point within the porcine kidney during HIFU sonication with (top) and without (middle) beam steering. The kidney displacement in the SI direction obtained from the fatselective navigator data is also shown (bottom). The temperature in the target point of the kidney was obtained from 3D motion compensated temperature maps. 


\title{
Spectrally selective pencil-beam navigator for motion compensation of MR- guided high-intensity focused ultrasound therapy of abdominal organs
}

\author{
Max O. Köhler ${ }^{1}$, Baudouin Denis de Senneville ${ }^{2}$, Bruno Quesson ${ }^{2}$, Chrit T.W. Moonen ${ }^{2}$, Mario \\ Ries $^{2}$ \\ ${ }^{1}$ Philips Healthcare, Finland, ${ }^{2}$ Laboratory for Molecular and Functional Imaging: From Physiology to \\ Therapy, University of Bordeaux 2, France
}

\author{
Running head: Spectrally selective pencil-beam navigator \\ Max Köhler \\ Address: Äyritie 4, 01510 Vantaa, Finland \\ Tel: +358 504151398 \\ Email: max.kohler@philips.com \\ Word count: 5176
}

Magnetic Resonance in Medicine 


\begin{abstract}
MR-guided high-intensity focused ultrasound (MR-HIFU) is a noninvasive technique for depositing thermal energy in a controlled manner deep within the body. However, the MR-HIFU treatment of mobile abdominal organs is problematic since motion-related thermometry artifacts need to be corrected and the focal point position must be updated to follow the moving organ in order to avoid damaging healthy tissue. In this paper, a fat-selective pencil-beam navigator is proposed for real-time monitoring and compensation of through-plane motion. As opposed to the conventional spectrally non-selective navigator, the fat-selective navigator does not perturb the water-proton magnetization used for proton resonance frequency shift thermometry. This allows the proposed navigator to be placed directly on the target organ for improved motion estimation accuracy. The spectral and spatial selectivity of the proposed navigator pulse is evaluated through simulations and experiments, and the improved slice tracking performance is demonstrated in vivo by tracking experiments on a human kidney and on a human liver. The direct motion estimation provided by the fat-selective navigator is also shown to enable accurate motion compensated MR-HIFU therapy of in vivo porcine kidney, including motion compensation of thermometry and beam steering based on the observed three-dimensional kidney motion.
\end{abstract}

Keywords: HIFU, MR thermometry, pencil beam, navigator, spectrally selective, beam steering 


\section{Introduction}

High-intensity focused ultrasound (HIFU) is a noninvasive technique for performing local thermal therapy $(1,2)$. The combination of MRI with HIFU (MR-HIFU) for providing image guidance is particularly promising since the excellent soft-tissue contrast of MRI allows for exact target localization and evaluation of treatment outcome $(3,4)$.

The main utility of MRI for HIFU therapy is, however, its ability to produce real-time maps of the instantaneous in vivo temperature distribution in any imaging plane with a high spatial resolution. Temperature images can be obtained by utilizing the linear temperature dependency of the proton resonance frequency (PRF) shift of water and the change in MR phase information between sequentially acquired gradient-echo images $(5,6)$. The relationship between the PRF shift and temperature is nearly independent of tissue type with the exception of adipose tissue whose PRF is almost constant despite variations in temperature $(7,8)$. Consequently, fat suppression or water-selective excitation is required in order to avoid errors in the PRF-based temperature images $(9,10)$.

Several abdominal organs would be prime candidates for MR-HIFU therapy, but are unfortunately subject to rigid or non-rigid motion during the respiratory cycle. This leads to two problems. First, the respiration-induced organ displacement and deformation will modify the local field and thus also the local magnetic field experienced by the target organ. This will result in phase variations and thus temperature Comment [f1]: R1.1 artifacts. Second, the focal point of the HIFU-system must also be adaptively repositioned as the organ moves with respect to the external transducer in order to avoid damaging healthy tissue and ensure an efficient heating.

Several approaches for the removal of thermometry artifacts have been proposed in the pas multi-baseline corrections to sample periodic changes $(11,12)$ and referenceless phase corrections $(13)$. A recent approach combined multi-baseline corrections with rapid 2D image registration to provide both a removal of thermometry artifacts and a correction of in-plane displacement in real-time (14). Slice tracking based on pencil-beam navigator data can moreover be used to address the remaining throughplane motion $(15,16)$, thus allowing the motion compensation to be extended to three dimensions. This approach was recently shown to enable real-time 3D motion compensated MR-HIFU therapy, including real-time 3D motion compensated MR thermometry and real-time beam steering of the HIFU focal spot according to the obtained 3D motion of the target region (17). 
The conventional pencil-beam navigator is, however, not well-suited for providing the exact motion estimation that is imperative for safe MR-HIFU therapy of mobile organs. For MR-HIFU, the navigator should preferably be placed directly on the target tissue for an exact motion compensation of both thermometry and focal point position. The pencil-beam navigator will thus necessarily intersect the imaging slice or slices that monitor the temperature rise in the target region when used for through-plane motion compensation. Moreover, both the conventional pencil-beam navigator and the imaging slices used for PRF-based thermometry act on the same water-proton magnetization. The navigator will thus perturb the magnetization of the imaging slice and vice versa, which may lead to reduced temperature accuracy, temperature artifacts, and slice tracking errors. The conventional proton-density weighted pencil-beam navigator will also suffer from poor contrast in the abdominal region that may further degrade the tracking performance.

Here, a spectrally selective pencil-beam navigator is presented. A fat-selective pencil-beam navigator should not perturb the water-proton magnetization of the imaging slice, or vice versa, and may thus provide an elegant solution to the problems of the conventional pencil-beam navigator for use in MRHIFU. The proposed RF pulse was evaluated theoretically as well as experimentally. In vivo experiments were performed to assess the slice tracking capabilities of the proposed navigator when placed directly on the kidney and on the liver of a healthy volunteer. Moreover, the efficacy of the fat-selective navigator for providing the accurate through-plane motion estimate necessary for 3D motion compensated MR-HIFU, including compensation of thermometry and focal point position, was evaluated in vivo on the kidney of a pig.

\section{Theory}

Similar to 2D acquisition schemes, such as spiral acquisition, 2D spatial selective excitation can be constructed by coupling an RF waveform with a $2 \mathrm{D}$ gradient waveform that determines the k-space trajectory of the excitation $(18,19)$. Nehrke et al. (16) proposed such an excitation utilizing a spiral kspace trajectory in conjunction with a $1 \mathrm{D}$ selective readout for achieving a pencil-beam shaped navigator echo, further improving the approach initially presented by Hardy et al.(20).

Compared to other k-space trajectories, an inward spiral k-space trajectory for 2D RF excitation shows several interesting properties. Most notably, the $2 \mathrm{D}$ RF pulse will be inherently self-refocused as the inward spiral trajectory ends in the k-space center (18). The spiral can also be considered to cover the kspace symmetrically, resulting in a linear dependence between the transversal magnetization and the 
applied $B_{1}$ field (19). The spiral trajectory will, moreover, utilize the gradient system very efficiently and thus allows short pulse durations to encode the desired part of $\mathrm{k}$-space.

However, the limitations of the available encoding time and the maximal gradient strength will introduce a circular aperture in k-space within which the radial k-space coverage is discrete. Consequently, the central peak of the spiral excitation point spread function (PSF) has the form of a jinc function surrounded by a series of regularly spaced radial secondary lobes that are interspersed by small amplitude oscillations (21). The first of the evenly spaced radial secondary lobes can be found at

$r_{1}=\frac{2 \pi N}{k_{\max }}=\frac{2 \pi N}{3.83} r_{0}$

where $N$ is the amount of $\mathrm{k}$-space spiral turns, and $r_{0}$ is the pencil-beam radius that is here defined as the first zero-crossing of the jinc-shaped PSF center peak (16). The amplitude of the oscillations can be attenuated by a suitable apodization function at the expense of a slightly broadened central peak (16). For this purpose, the desired excitation profile is here chosen to be that of a disk with radius equal to the pencil beam. The convolution of this disk-shaped desired excitation profile with the PSF will determine the actual excitation profile, thereby dampening the oscillations between the side lobes of the PSF.

The disadvantages of the conventional pencil-beam excitation for motion compensation of MR-HIFU largely arise because the pencil beam excites the same water-proton magnetization as the imaging slices used for PRF-thermometry. Spectrally selective excitation offers a potentially elegant solution to this problem (10). Water-selective excitation may be used to discard the fat as is necessary for PRF-based thermometry to avoid erroneous temperature readings. A fat-selective pencil beam would therefore avoid perturbing the magnetization in the imaging slice, and vice versa. Furthermore, a spectrally selective pencil beam should provide an improved contrast as compared to a conventional spectrally non-selective pencil beam since most abdominal organs are surrounded by a sheath of fat.

Binomial weighting of the 2D RF pulses can be used to provide the desired spectral selectivity with the prerequisite that the transverse magnetization is refocused between the individual pulses $(22,23)$. The separation in time between the binomial pulses must allow fat and water to reach opposite phase. A phase condition of $1 / 2 \pi$ is usually chosen as this makes the spectral selectivity less sensitive to $B_{0}$ inhomogeneities. However, for the case of binomially weighted 2D RF pulses this is not feasible since the maximum gradient strength and gradient slew rates of most whole-body MR scanners would not allow time for sufficient spatial encoding of the excitation despite the use of spiral k-space trajectories. On a 1.5 
T MR scanner with gradient strength of $21 \mathrm{mT} / \mathrm{m}$ and slew rate of $100 \mathrm{~T} / \mathrm{m} / \mathrm{s}$ as used in this study, the maximum amount of spiral turns would be restricted to 2 if a phase separation of $1 / 2 \pi$ is desired. This would result in an extremely poorly shaped PSF with densely spaced radial side lobes of high amplitude $(16,21)$. The second best alternative is then to opt for a fat-water phase difference of $3 / 2 \pi$. Although the longer fat-water dephasing will degrade the ability of the binomial pulse train to separate water and fat, it does allow for a 12-turn spiral trajectory excitation. This provides a sufficiently dense radial coverage of the k-space to produce a well-defined PSF with a sharp central peak and only a few aliasing rings of small amplitudes situated far from the central peak (16).

Even though individual inward spiral trajectories are inherently self-refocused, gradients need to be applied prior to every 2D RF pulse following the first pulse of the pulse train in order to obtain the same starting point of the k-space trajectory for each of the 2D RF pulses. The k-space starting point of each spiral trajectory could alternatively be slightly rotated to reduce the peak amplitude of the side lobes (24). This is, however, of limited interest for a train of 12-turn spirals whose first radial side lobe occurs at a distance approximately 20 times the radius of the pencil beam. Furthermore, rotation of the k-space starting point would require a different gradient waveform for each 2D RF pulse, which might make the pulse train more sensitive to eddy currents and $B_{1}$ inhomogeneities $(23,25)$.

Although longer binomial pulse trains provide an improved spectral selectivity with a wider stopband (26), motion compensated MR-HIFU therapy of mobile organs imposes strict real-time constraints. The pulse train used in this paper is therefore limited to three binomially weighted 2D RF pulses as a compromise between the requirements on temporal resolution and spectral selectivity. The sequence diagram of the proposed 2D RF pulse train is shown in Fig. 1. The individual 2D RF pulses of the pulse train use a 12-turn spiral k-space trajectory and consecutive pulses are separated by an interval of $6.91 \mathrm{~ms}$ corresponding to a fat-water phase difference of $3 / 2 \pi$ at $1.5 \mathrm{~T}$. This gives rise to a total duration of 19.9 ms for the 1-2-1 binomially weighted 2D RF pulse train. Pulse trains of 2D RF pulses based on spiral trajectories have previously been proposed for excitation of three dimensional volumes (24) and for compensation of field inhomogeneities (27).

\section{Materials \& Methods}

\section{Simulations}

The theoretical performance of the proposed 1-2-1 binomial pencil-beam excitation was evaluated using Bloch-simulation software (http://www-mrsrl.stanford.edu/ brian/bloch/) ported to IDL 6.1.1 (ITT Corp., 
White Plaines, NY). The diameter of the simulated pencil beam was $30 \mathrm{~mm}$ and the total flip angle of the pulse train was $25^{\circ}$. Relaxation was omitted from the simulations.

\section{MR imaging}

The spectrally selective 1-2-1 binomial pulse train of 2D spiral excitations was implemented on a $1.5 \mathrm{~T}$ Philips Achieva scanner (Philips Healthcare, Best, The Netherlands). The implementation was based on the existing implementation of the spiral encoded pencil-beam navigator that is corrected for nonuniform sampling density and short-term eddy currents (16). The intended diameter of the pencil-beam navigator was $30 \mathrm{~mm}$ and the total flip angle of the pulse train was $25^{\circ}$.

The excitation profile of the binomial spiral excitation pulse train was obtained by using this 2D RF pulse train as an excitation pulse coupled with a turbo spin echo $(\mathrm{TSE})$ readout $(\mathrm{TR}=1000 \mathrm{~ms}, \mathrm{TE}=58 \mathrm{~ms}$, FOV $=300 \times 300 \mathrm{~mm}^{2}$, matrix $=128 \times 128$, slice thickness $=7 \mathrm{~mm}$ ), where the refocusing pulse was made slice selective perpendicularly to the pencil beam axis. The target was a homogenous manganesechloride doped phantom.

The slice tracking capabilities of the proposed fat-selective navigator were evaluated in vivo by comparing the positional data obtained with the spectrally selective navigator to that of the non-selective 12-turn spiral navigator when imaging a human kidney and a human liver under free breathing. The experiments were performed on two separate volunteers from whom informed consent was obtained. An axial imaging slice was chosen and the navigator was placed perpendicularly to the imaging slice in the superior-inferior (SI) direction across the fat capsule at the lower apex of the right kidney in the kidney experiment, and across the gallbladder and the surrounding fat in the liver experiment. The positioning of the imaging slice and the navigator for the kidney and the liver experiment is shown in Fig. 2. The sequence used for imaging in both experiments was a single-shot gradient-echo echo planar imaging $(\mathrm{EPI})$ sequence with TR $=110 \mathrm{~ms}, \mathrm{TE}=48 \mathrm{~ms}$, flip angle $=35^{\circ}, \mathrm{FOV}=300 \times 195 \mathrm{~mm}^{2}$, matrix $=128 \times$ 83 , slice thickness $=6 \mathrm{~mm}$, and a 1-2-1 binomial water-selective excitation pulse. One-dimensional navigator profiles were acquired before each dynamic repetition of the imaging sequence by performing a readout along the axis of the excited pencil beam. The imaging slice position was adaptively corrected in real-time based on the estimated target displacement as obtained via correlation analysis of subsequently acquired navigator profiles $(15,28)$. The duration of the single non-selective $2 \mathrm{D}$ RF pulse was $6.1 \mathrm{~ms}$, whereas the duration of the fat-selective 2D RF pulse train was $19.9 \mathrm{~ms}$. The duration of the 1D readout 
was in turn $6.6 \mathrm{~ms}$, resulting in a total duration of 12.7 and $26.5 \mathrm{~ms}$ for the non-selective and fat-selective navigators, respectively.

\section{MR-HIFU platform}

The MR-HIFU experiment was performed on a Sonalleve MR-HIFU platform (Philips Healthcare, Vantaa, Finland) that was integrated into the 1.5 T Philips Achieva MR scanner. The MR-HIFU platform consisted of a HIFU transducer embedded in a water-filled MR table top, an RF generator, and a workstation dedicated to therapy control. The 256 element HIFU transducer (Imasonic, Voray sur l'Ognon, France) had spherical shell geometry with a focal length of $12 \mathrm{~cm}$ and aperture of $13 \mathrm{~cm}$, and was operated at 1.2 MHz. The measured focal point size was $1 \times 1 \times 7 \mathrm{~mm}^{3}$. More details on the MRHIFU platform can be found in Ref. (29).

\section{MR thermometry}

The temperature rise induced by the HIFU ablation of the in vivo porcine kidney was monitored in a single axial slice with the proposed fat-selective navigator placed perpendicularly to the imaging slice across the fat capsule of the kidney. The dynamic images used for MR thermometry were acquired with a single-shot gradient-echo EPI sequence with the following acquisition parameters: $\mathrm{TR}=100 \mathrm{~ms}$, $\mathrm{TE}=48$ ms, flip angle $=35^{\circ}, \mathrm{FOV}=300 \times 195 \mathrm{~mm}^{2}$, matrix $=128 \times 83$, and slice thickness $=6 \mathrm{~mm}$. A 1-2-1 binomial water-selective RF pulse was used to suppress the signal of the fat and avoid thermometry errors. The imaging slice position was adaptively corrected in real-time by modifying the excitation frequency of the water-selective RF pulse according to the estimated kidney motion obtained via correlation analysis of the navigator profiles $(15,28)$.

The acquired images were in-plane realigned prior to the calculation of temperature maps as described in Ref. (14). The principle direction of kidney respiratory motion is the SI direction, which was addressed by slice tracking using the fat-selective navigator. The combination of the estimated 2D in-plane motion with the navigator-based estimate of the through-plane motion provided the 3D motion estimate necessary for fixing the focal point within the moving kidney as described in Ref. (17). The remaining periodic phase fluctuations due to respiratory motion were corrected using a multi-baseline approach where the look-uptable was indexed according to the kidney displacement estimate obtained by the navigator as explained by Ries et al. (17). The calculation of the PRF-based temperature maps and the employed corrections are described in detail by Roujol et al. (14). 
In vivo three-dimensional motion compensated MR-HIFU

The efficacy of the fat-selective navigator for accurately estimating the through-plane motion required for 3D motion compensated MR-HIFU was evaluated on in vivo porcine kidney. The animal (45 $\mathrm{kg}$ ) was initially anesthetized with a premedication of $10 \mathrm{ml}$ of propofol. General anesthesia was induced and maintained during the experiment with a continuous intravenous infusion of propofol at $1 \mathrm{ml} / \mathrm{min}$. The animal was intubated and ventilated (45 to $100 \%$ O2, respirator paraPac, ResMed SA, France) with a respiratory cycle of approximately $3 \mathrm{~s}$ during the entire procedure. The animal study was performed with the approval of the local ethics committee.

The animal was positioned in a decubitus position on the MR-HIFU table top with the targeted kidney centered on the acoustic window. Following the positioning of the animal, HIFU ablation of the kidney was performed in a single focal point that was steered in real-time according to the estimated 3D motion of the targeted kidney (17). The asymmetric transducer design allowed the electronically steered focal spot to follow the $8 \mathrm{~mm}$ peak-to-peak respiratory motion of the porcine kidney without generating significant secondary lobes. The update of the focal point position was performed synchronously to MRimaging at a frequency of $10 \mathrm{~Hz}$. The ablation was performed using a constant power of $100 \mathrm{~W}$ and sonication duration of $30 \mathrm{~s}$.

An in-house developed real-time reconstructor was used to reduce the time required for MR image reconstruction. The time needed for acquisition (after passage of the k-space center) and data transport (64 ms), image reconstruction and motion estimation $(40 \mathrm{~ms})$, and HIFU focal point update (10 ms) resulted in a focal point position update latency of $114 \mathrm{~ms}$. Details on the employed beam steering strategy, including the required calculations and their timings, are given by Ries et al. (17).

\section{Results}

\section{Simulation}

The spectral selectivity of the proposed navigator was demonstrated through simulations at different frequency offsets, where the resulting signal was taken as the complex sum of the transverse magnetization over the 15-mm radius disk intended for excitation. The results are shown in Fig. 3 for both the fat- and water-selective pencil-beam excitations. For comparison, the spectral response of the conventional non-selective pencil-beam excitation at the resonance frequency of water and fat is also included. Note that the peak-to-valley separation in the spectral response of the binomial pencil-beam 
excitation was $72 \mathrm{~Hz}$, corresponding to one third of the $217 \mathrm{~Hz}$ fat-water resonance frequency shift at $1.5 \mathrm{~T}$. This is a direct consequence of using a fat-water phase difference of $3 / 2 \pi$ instead of the $1 / 2 \pi$ commonly used.

Even though there were additional spectral response peaks outside the desired on-resonance response, the spatial selectivity of the binomially weighted $2 \mathrm{D}$ RF pulse train rapidly decreased with increasing frequency offset. This can be seen in Fig. 4 where the excitation profiles at on-resonance and at the first two off-resonance spectral peaks are shown. However, when placing the navigator directly on the abdominal target organ, frequency offsets of approximately $1 \mathrm{ppm}(64 \mathrm{~Hz}$ at $1.5 \mathrm{~T})$ can be expected, i.e. far less than the $2.3 \mathrm{ppm}(145 \mathrm{~Hz})$ frequency offset of the first spectral side peak. For such frequency offsets of around $1 \mathrm{ppm}$, the central peak of the excitation profile had a full width at half maximum (FWHM) within 6\% of the $22.9 \mathrm{~mm}$ on-resonance $\mathrm{Fr}^{\mathrm{T}}$. Further off-resonance, the spatial selectivity quickly degenerated to a FWHM of $73 \mathrm{~mm}$ at $3 \mathrm{pp}$ even larger frequency offsets, the increased blurring of the central peak caused the magnetization maximum to be located away from the pencil beam axis as can be seen to occur for the excitation profile at the second off-resonance spectral peak in Fig. 4.

The excitation profile central peak of the binomially weighted 2D RF pulse train coincided with that of the conventional spectrally non-selective single $2 \mathrm{D}$ RF pulse at the frequency offsets corresponding to the spectral maxima seen in Fig. 3. Outside these spectral maxima, the excitation profiles of the spectralselective and non-selective pencil-beam excitations did differ. Moreover, according to Eq. 1 the first ring lobes are to be expected at $295 \mathrm{~mm}$ off-axis for a conventional 12-turn spiral encoded 2D RF pulse with an intended pencil beam diameter of $30 \mathrm{~mm}$. Figure 4 shows that this is also the case when the spiral encoded 2D RF pulse is applied in a binomial pulse train at on-resonance.

\section{On-resonance excitation profile comparison}

The central peak of the simulated and experimental excitation profiles are compared in Fig. 5 for the proposed 1-2-1 binomial 2D RF pulse train. Within the limits imposed by the SNR and the imperfect $B_{0}$ shim, the measured excitation profile central peak matched the predicted simulated result of a jinc function with a FWHM of $22.1 \mathrm{~mm}$.

The small oscillations surrounding the central peak deviated somewhat from the ideal radial form in the experimental data by showing slightly larger amplitude in the negative readout direction than in the phase-encoding and positive readout direction. This effect can be attributed to small deviations from the ideal spiral trajectory due to minor gradient system imperfections. 
1

2

3

4

\section{In vivo imaging}

Figure 6a shows a comparison of a slice tracking experiment on a human kidney using a spectrally nonselective conventional pencil-beam navigator and the proposed fat-selective pencil-beam navigator. Figure $6 \mathrm{~b}$ shows a similar comparison of slice tracking experiments using the two navigators on a human liver. In both the kidney and the liver experiment, the spectrally selective navigator showed a good contrast-to-noise ratio and was successful in tracking the organ position over the entire scan duration (1500 images, 3 min scanning, frame-rate 9 images/s) without apparent tracking errors. Although the conventional navigator did have higher SNR, the lower contrast-to-noise ratio caused problems for the correlation algorithm used to determine the target displacement. Moreover, the onset of water-selective MR imaging perturbed the water-magnetization within the non-selective pencil beam in both the kidney and the liver experiment, thus altering the acquired navigator profile (indicated by arrow in Fig. 6). Since the resulting partial saturation was the dominant feature within the non-selective navigator profile in the kidney experiment (Fig. 6a), the navigator-based motion estimation did not follow the kidney motion at all but instead tracked the position of the previously excited imaging plane. As a consequence, the kidney motion was consistently underestimated as can seen by comparing the amplitude of the displacement estimate with the motion of the darker grey band in the middle of the navigator profile. The partial saturation caused by the imaging slice was less dominant in the non-selective navigator profile of the liver experiment (Fig. 6b) due to the higher contrast. Consequently, the motion estimation based on the nonselective navigator was successful during most of the liver experiment. However, it failed to follow the motion of the liver through the entire scan, particularly towards the end of the scan where the motion estimate was sporadic. This can be appreciated by noting that the red displacement estimate does not match the smooth motion of the navigator profile features (e.g. the motion of the fat that appears dark at the bottom of the profile).

In theory, there should be no alteration of the navigator profile at onset of the MR imaging for the fatselective navigator, although in practice a very slight alteration could be seen in both the kidney and liver experiments that can be attributed to off-resonance effects. The magnitude of the alteration was, however, insignificant and did not have any effect on the tracking performance in either experiment. The barely visible alteration of the navigator profile at onset of imaging indicates that the water-selective MR imaging does not notably affect the lipid-proton magnetization excited by the fat-selective navigator. The lower SNR of the fat-selective navigator did, however, result in a slight jitter in the motion estimate.

In vivo motion compensated MR-HIFU 
Figure 7 shows the 3D motion compensated temperature map obtained at the end of a $30 \mathrm{~s}$ beam-steered HIFU sonication on in vivo porcine kidney. The temporal evolution of the temperature at the target point within the kidney can in turn be seen in Fig. 8 for both the beam steered sonication and for a stationary HIFU sonication. Figure 8 also shows the kidney displacement in the SI direction as obtained from the proposed fat-selective navigator that was used for both slice tracking and beam steering. The peak temperature for the stationary HIFU sonication experiment was found to be $6.0{ }^{\circ} \mathrm{C}$ and thus $25 \%$ lower than the peak value of $8.1^{\circ} \mathrm{C}$ for the beam steered experiment. The fat-selective navigator enabled the slice and the HIFU-beam to remain locked on the target area for the entire duration of the experiment (5 $\min )$. No interference between the fat-selective navigator beam and the water-selective imaging slice was observed.

A similar 3D motion compensated experiment was attempted with the non-selective navigator, but in this case the navigator failed to reliably represent the motion pattern of the kidney. Since the correct measurement of the organ displacement is indispensable for multi-baseline thermometry correction, this rendered thermometry impossible and no HIFU ablation was attempted.

\section{Discussion}

In this study, a spectrally selective pencil-beam navigator pulse was designed for direct motion tracking of mobile abdominal organs that are surrounded by islets of fat, such as the kidney or the liver. For such organs, a fat-selective navigator can provide positional information with high contrast-to-noise without interfering with water-selective MR imaging and vice versa. This enables the proposed navigator to be placed directly on the organ of interest for improved accuracy in the monitoring of the organ motion, instead of indirectly estimating the organ motion based on the observed movement of the diaphragm as is typically done with conventional navigators.

However, spiral encoded 2D spatial selective excitation and frequency selective binomial excitation are both limited by their off-resonance sensitivity when used separately. Hence, a careful analysis of the limitations of the combination of the two methods has to be carried out.

\section{Off-resonance sensitivity of spectral response}

The spectral response of the proposed binomial 2D RF pulse train is by design similar to the original 1-21 binomial 1D RF pulse train $(22,23)$ as seen in Fig. 3. However, the original $1 / 2 \pi$ fat-water phase condition was relaxed to $3 / 2 \pi$ to allow for a sufficiently dense coverage of excitation k-space to create a 
well-defined PSF with only a few sparse low-amplitude aliasing rings and a sharp central peak. This relaxation of the phase condition caused additional spectral response zeroes to occur at one third of the fat-water frequency shift above the frequency of each spectral peak as seen in Fig. 3. Consequently, the width of spectral peaks and valleys is decreased, thereby making the spectral selectivity of the proposed 1-2-1 binomial 2D RF pulse train more sensitive to field inhomogeneities than the original 1-2-1 binomial pulse train. For the conventional binomial 1D RF pulse train (data not shown), a deviation of $0.69 \mathrm{ppm}$ from on-resonance can be tolerated while still keeping the signal of the undesired spectral component (e.g. water) below $10 \%$ of maximum. By comparison, an off-resonance shift of $0.34 \mathrm{ppm}$ will provide a similar signal level from the undesired frequency band for the binomial 2D RF pulse train. The field homogeneity should thus be approximately twice as good for the binomial 1-2-1 2D RF pulse train as for the conventional 1-2-1 binomial pulse in order to give a comparable suppression of the undesired spectral component, with the exact ratio depending on the required level of suppression.

The main contributing factor to the increased off-resonance sensitivity of the binomial 2D RF pulse train spectral response is thus the relaxed phase condition. The spectral selectivity is also, although to a far lesser extent, affected by the off-resonance blurring of the 2D RF pulse spatial profile that alters the offresonance spectral peaks and indirectly affects the bandwidth of the spectral stopband.

\section{Off-resonance sensitivity of spatial response}

The on-resonance spatial response of the proposed 2D RF pulse train is not affected by the binomial weighting of the pulse train. Consequently, the on-resonance excitation profile had a sharp central peak with a FWHM of $22.9 \mathrm{~mm}$ and sparse radial side lobes of low amplitude as seen in Fig. 4 and 5. The first radial side lobe was situated at approximately $30 \mathrm{~cm}$ from the beam axis, thus having only a minor effect on the obtained navigator profile.

At off-resonance frequencies, the evaluation of the spatial selectivity is complicated by that the fact that the PSF of a 2D spatially selective excitation pulse is in general frequency dependent and that this spatial response is furthermore weighted by the binomial pulse train. However, for the proposed RF pulse, the binomial pulse train merely provides a spectral weighting of the spectral-spatial response of the individual 2D RF pulses. Consequently, the spectral response of the binomial 2D RF pulse train is modulated by the spectral response of the individual 2D RF pulses, and the two spectral response curves therefore coincide at the spectral maxima of the binomial 2D RF pulse as seen in Fig. 3. At the corresponding frequency offsets, the blurred off-resonance excitation profile (see Fig. 4) proved to be identical for the single 2D RF pulse and for the binomial pulse train of $2 \mathrm{D}$ RF pulses. 
The spatial response of the single 12-turn spiral excitation pulse and the binomial pulse train of 12-turn spiral excitations did, however, differ outside these spectral maxima due to the binomial weighting. At 1 ppm off-resonance, the FWHM of the excitation profile central peak was $24.0 \mathrm{~mm}$ for the single 2D RF pulse, whereas it was further degraded to $24.2 \mathrm{~mm}$ for the binomial 2D RF pulse train. The influence of the binomial weighting on the excitation profile of the 2D RF pulse train was less significant further offresonance and the spatial selectivity of both the single 2D RF pulse and binomial 2D RF pulse train dropped rapidly. At the first spectral side peak of $2.7 \mathrm{ppm}(145 \mathrm{~Hz})$ the FWHM was broadened by $96 \%$, whereas at the second spectral side peak of $4.5 \mathrm{ppm}(290 \mathrm{~Hz})$ the maximum of the spatial response was no longer located in the desired beam area but shifted outwards toward an aliasing ring.

The binomial weighting only causes a slight further degradation of the spatial profile as compared to the single spiral excitation 2D RF pulse. Severely off-resonant tissue will be poorly localized for both the conventional and the spectrally selective pencil-beam excitation, and any resulting signal will enter the background noise of the navigator profile.

Sensitivity to gradient and $B_{1}$ imperfections

The high slew rates used by the spiral k-space encoding make the 2D RF pulses particularly sensitive to eddy currents (30). These eddy currents may distort the actual k-space trajectory from its nominal path, thereby causing the RF energy deposition to be misplaced within the excitation k-space. This may in turn degrade the excitation profile. However, no such degradation could be noticed between the theoretical and experimental excitation profile of the spiral 2D RF pulse train in Fig. 5, indicating that the precompensation of the gradient waveforms was successful in correcting for the $\mathrm{k}$-space distortion caused by the eddy currents (16).

Even if no compensation of the eddy currents would have been performed, the identical k-space trajectories and gradient waveforms of the individual 2D RF pulses will induce similar eddy currents for each pulse in the pulse train (25). This should in turn render the spectral selectivity of the proposed binomial 2D RF pulse train insensitive to eddy currents. The symmetrical and identical coverage of excitation k-space by the individual 2D RF pulses in the binomial pulse train also ensures that the spectral response of the proposed pulse is insensitive to $B_{1}$ inhomogeneities (23).

Motion estimation and compensation 
1

2

3

4

The main disadvantage of the proposed navigator is the time needed for excitation as compared to the original 2D navigator pulse of Nehrke et al. (16). A single 2D RF pulse only required $6.1 \mathrm{~ms}$ for encoding the excitation k-space with a 12-turn spiral, whereas applying this same spiral excitation in a 1-2-1 binomially weighted succession including the appropriate delays required $19.9 \mathrm{~ms}$. Only minor motion is nevertheless likely to occur during the $26.5 \mathrm{~ms}$ required for acquiring the fat-selective navigator echo. Even if assuming a sinusoidal target organ motion with a short breathing cycle of $4 \mathrm{~s}$ and a large motion amplitude of $25 \mathrm{~mm}$ as might occur in the upper abdomen, the organ motion will still only be $0.52 \mathrm{~mm}$ during the fat-selective navigator acquisition. This can be compared to an organ motion of $0.25 \mathrm{~mm}$ during the non-selective navigator acquisition $(12.7 \mathrm{~ms})$. The effect of the target motion on the motion estimate and the slice tracking performance should thus remain negligible for the spectral selective navigator.

The proposed navigator showed an excellent real-time tracking performance of respiration-induced abdominal motion for frame-rates up to 9 images per second as seen in Fig. 6 . The navigator profile of the fat-selective navigator displayed an improved contrast within the abdominal region as compared to the conventional navigator. Even though there should in theory be no interference between the fat-selective navigator and water-proton MR imaging, slight interferences due to off-resonance can occur in practice as seen in Fig. 6. The effect of the interference on the fat-selective navigator profile was, however, barely noticeable and thus had no influence on the tracking algorithm. This is in stark contrast to the conventional non-selective navigator where the partial saturation induced by the MR imaging was a dominating feature within the navigator profile causing severe problems for the motion estimation algorithm. These properties enabled the fat-selective navigator to be placed directly on the abdominal target organ for accurate monitoring of the organ motion without interfering with water-proton MR imaging. For large non-rigid organs such as the liver, the navigator should moreover be placed in the vicinity of the target volume in order for the motion registered by the navigator to correspond to that of the target.

The utility of the proposed navigator for direct and accurate monitoring of the target organ motion was shown with a three-dimensionally motion compensated MR-HIFU sonication on in vivo porcine kidney, where both thermometry and sonication were compensated based on the observed motion. For the motion compensation of MR thermometry, the fat-selective navigator was used for slice tracking as well as providing the index for the look-up-table based multi-baseline compensation. The navigator did not perturb the water-proton magnetization used for PRF-thermometry despite intersecting the imaging slice at the targeted kidney. When only thermometry was motion compensated and no beam steering was performed, the peak target temperature rise was $25 \%$ less than when beam steering was utilized. The 
somewhat small effect can be largely attributed to the mechanical ventilation, which caused the kidney to remain in place for most of the respiratory cycle as seen in Fig. 8. Moreover, the unnaturally rapid kidney motion at exhalation and inhalation enhanced the influence of the focal point update latency. Consequently, the effect of beam steering is likely to be substantially larger during free breathing than mechanical ventilation due to the smooth and continuous organ motion.

The experimental results focused on the fat-selective version of the navigator as it allows for the positioning of the navigator in the field of view of the MR imaging sequence without disturbing the water-proton magnetization, something that the spectrally non-selective and water-selective pencil-beam navigator do not. Moreover, the partial saturation of the non-selective navigator profile caused by the imaging slice may even cause the navigator to track the previously excited imaging plane instead of the organ motion, particularly if it is the dominating feature within the navigator profile as was seen in Fig. 6. A potential disadvantage of the proposed fat-selective navigator arises for lean patients with little fat around their abdominal organs. In such cases, however, a preceding fat-selective image may generally provide a suitable islet of fatty tissue in the vicinity of the organ to place the navigator beam on.

\section{Conclusions}

A spectrally selective navigator was presented for compensation of abdominal organ motion. Robust spectral and 2D spatial selectivity was achieved by combining three pencil-beam excitations into a 1-2-1 binomial pulse train. The resulting spectral selectivity allowed the fat-selective version of the navigator to be placed within the field of view of the MR sequence without interfering with water-proton based images. Direct tracking of the moving organ was achieved with excellent tracking performance due to the reduced interference of the water-proton imaging and the improved contrast-to-noise ratio of the navigator profile. The low interference of the fat-selective navigator on the water-signal, and vice versa, makes it ideal for direct and accurate tracking of organs as well as for PRF based thermometry experiments on mobile organs, where the fat-signal is typically discarded. The fat-selective navigator was also shown to enable accurate real-time motion compensation of MR-HIFU in vivo, where thermometry was compensated for three-dimensional motion and the focal point steered in real-time according to the observed motion.

\section{Acknowledgements}

The authors would like to thank Erkki Vahala of Philips Healthcare for helpful ideas and support on software development. 


\section{References}

1. Fry FJ. Precision high intensity focusing ultrasonic machines for surgery. Am J Phys Med 1958;37(3):152-156.

2. Tempany CM, Stewart EA, McDannold N, Quade BJ, Jolesz FA, Hynynen K. MR imaging-guided focused ultrasound surgery of uterine leiomyomas: a feasibility study. Radiology 2003;226(3):897905.

3. Cline HE, Schenck JF, Hynynen K, Watkins RD, Souza SP, Jolesz FA. MR-guided focused ultrasound surgery. J Comput Assist Tomogr 1992;16(6):956-965.

4. Hynynen K, Freund WR, Cline HE, Chung AH, Watkins RD, Vetro JP, Jolesz FA. A clinical, noninvasive, MR imaging-monitored ultrasound surgery method. Radiographics 1996;16(1):185195.

5. Hindman JC. Proton resonance shift of water in gas and liquid states. J Chem Phys 1966;44:45824592.

6. Ishihara Y, Calderon A, Watanabe H, Okamoto K, Suzuki Y, Kuroda K, Suzuki Y. A precise and fast temperature mapping using water proton chemical shift. Magn Reson Med 1995;34(6):814-823.

7. De Poorter J. Noninvasive MRI thermometry with the proton resonance frequency method: study of susceptibility effects. Magn Reson Med 1995;34(3):359-367.

8. Peters RD, Hinks RS, Henkelman RM. Ex vivo tissue-type independence in proton-resonance frequency shift MR thermometry. Magn Reson Med 1998;40(3):454-459.

9. de Zwart JA, Vimeux FC, Delalande C, Canioni P, Moonen CT. Fast lipid-suppressed MR temperature mapping with echo-shifted gradient-echo imaging and spectral-spatial excitation. Magn Reson Med 1999;42(1):53-59.

10. Meyer CH, Pauly JM, Macovski A, Nishimura DG. Simultaneous spatial and spectral selective excitation. Magn Reson Med 1990;15(2):287-304.

11. Denis de Senneville B, Mougenot C, Moonen CT. Real-time adaptive methods for treatment of mobile organs by MRI-controlled high-intensity focused ultrasound. Magn Reson Med 2007;57(2):319-330.

12. Vigen KK, Daniel BL, Pauly JM, Butts K. Triggered, navigated, multi-baseline method for proton resonance frequency temperature mapping with respiratory motion. Magn Reson Med 2003;50(5):1003-1010.

13. Rieke V, Vigen KK, Sommer G, Daniel BL, Pauly JM, Butts K. Referenceless PRF shift thermometry. Magn Reson Med 2004;51(6):1223-1231.

14. Roujol S, Ries M, Quesson B, Moonen C, Denis de SB. Real-time MR-thermometry and dosimetry for interventional guidance on abdominal organs. Magn Reson Med 2010;63(4):1080-1087. 
15. McConnell MV, Khasgiwala VC, Savord BJ, Chen MH, Chuang ML, Edelman RR, Manning WJ. Prospective adaptive navigator correction for breath-hold MR coronary angiography. Magn Reson Med 1997;37(1):148-152.

16. Nehrke K, Börnert P, Groen J, Smink J, Böck JC. On the performance and accuracy of 2D navigator pulses. Magn Reson Imaging 1999;17(8):1173-1181.

17. Ries M, Denis de Senneville B, Roujol S, Berber Y, Quesson B, Moonen C. Real-time 3D target tracking in MRI guided focused ultrasound ablation in moving tissues. Magn Reson Med 2010;in press.

18. Pauly J, Nishimura D, Macovski A. A k-space analysis of small-tip-angle excitation. J Magn Reson 1989;81:43-56.

19. Pauly J, Nishimura D, Macovski A. A linear class of large tip-angle selective excitation pulses. J Magn Reson 1989;82:571-587.

20. Hardy CJ, Pearlman JD, Moore JR, Roemer PB, Cline HE. Rapid NMR cardiography with a halfecho M-mode method. J Comput Assist Tomogr 1991;15(5):868-874.

21. Lauzon ML, Rutt BK. Effects of polar sampling in k-space. Magn Reson Med 1996;36(6):940-949.

22. Hore PJ. Solvent suppression in Fourier transform nuclear magnetic resonance. J Magn Reson 1983;55(2):283-301.

23. Schick F, Forster J, Machann J, Huppert P, Claussen CD. Highly selective water and fat imaging applying multislice sequences without sensitivity to B1 field inhomogeneities. Magn Reson Med 1997;38(2):269-274.

24. Pauly JM, Hu BS, Wang SJ, Nishimura DG, Macovski A. A three-dimensional spin-echo or inversion pulse. Magn Reson Med 1993;29(1):2-6.

25. Block W, Pauly J, Kerr A, Nishimura D. Consistent fat suppression with compensated spectralspatial pulses. Magn Reson Med 1997;38(2):198-206.

26. Schick F. Numerically optimized hard pulse sequences for fast frequency selective excitation and inversion. MAGMA 1993;1(3):158-168.

27. Morrell G, Macovski A. Three-dimensional spectral-spatial excitation. Magn Reson Med 1997;37(3):378-386.

28. Wang Y, Grimm RC, Felmlee JP, Riederer SJ, Ehman RL. Algorithms for extracting motion information from navigator echoes. Magn Reson Med 1996;36(1):117-123.

29. Köhler MO, Mougenot C, Quesson B, Enholm J, Le Bail B, Laurent C, Moonen CT, Ehnholm GJ. Volumetric HIFU ablation under 3D guidance of rapid MRI thermometry. Med Phys 2009;36(8):3521-3535.

30. Takahashi A, Peters T. Compensation of multi-dimensional selective excitation pulses using measured k-space trajectories. Magn Reson Med 1995;34(3):446-456. 
FIG. 1. Gradient and RF waveforms of the proposed 1-2-1 binomial 2D RF pulse train. The excitation kspace encoding is over 12 spiral turns, with a fat-water dephasing corresponding to $3 / 2 \pi$. The RF waveform is weighted according to the nonuniform sampling density.

FIG. 2. Position of navigator (black rectangle) and imaging slice (white rectangle) for the (a) kidney and (b) liver in vivo slice tracking experiments. The navigator pencil beam was placed across the lower apex of the right kidney in the kidney experiment. For the liver experiment, the navigator was placed across the gallbladder and the surrounding fat at the bottom of the liver. The navigators intersected the axial imaging slice perpendicularly in both experiments.

FIG. 3. Spectral response of the water-selective (dashed) and fat-selective (solid) pencil-beam navigator. The two envelopes (dotted) correspond to the non-selective navigator when excited at the fat and water resonance frequency. The distance between neighboring spectral peaks and valleys of $72 \mathrm{~Hz}$ is also shown.

FIG. 4. Excitation profile of the spectrally selective pencil-beam excitation at frequency offsets of $0 \mathrm{~Hz}$ (solid), $145 \mathrm{~Hz}$ (dotted) and $290 \mathrm{~Hz}$ (dashed). The excitation profiles of both the conventional nonselective and the spectral-selective pencil-beam excitation coincide at the frequency offsets corresponding to the spectral peaks of Fig. 3. The full width at half maximum (FWHM) of the excitation profiles at 0 and $145 \mathrm{~Hz}$ are also indicated.

FIG. 5. Comparison of the (a) theoretical and (b) experimental central peak of the on-resonance excitation profile as viewed perpendicularly to the pencil-beam direction. The excitation profile shows a good spatial localization with the central peak in the expected form of a jinc function.

FIG. 6. Comparison of the slice tracking performance of the conventional spectrally non-selective navigator (NS) and the fat-selective navigator (FS) when placed directly on (a) the kidney and (b) the liver. The navigator profiles are plotted vertically with subsequently acquired profiles stacked after each other and the dynamic scan number increasing towards the right. The displacements estimated based on the correlation of the navigator profiles are depicted in red. The navigator profile obtained by the conventional non-selective navigator is altered significantly by the onset of the water-selective MR imaging (indicated by arrow). This effect is much less pronounced for the fat-selective navigator profile. Note that the displacement estimates were shifted relative to the navigator profiles in order not to interfere with the essential features of the navigator profiles. 
FIG. 7. Temperature map of the kidney at the end of a beam-steered HIFU sonication. The motion compensated temperature map is overlaid in color on the axial magnitude image. The transducer and the semi-transpar am-path overlay as well as the location of the gel pad are shown to clarify the experimental setup. A close-up of the temperature map within the beam path is also shown (top) with the black arrowhead indicating the position of the focus within the kidney. Two additional hotspots (white arrowheads) due near-field heating can also be seen. The proposed fat-selective navigator was used to provide the necessary through-plane motion estimate.

FIG. 8. Temperature rise in the target point within the porcine kidney during HIFU sonication with (top) and without (middle) beam steering. The kidney displacement in the SI direction obtained from the fatselective navigator data is also shown (bottom). The temperature in the target point of the kidney was obtained from 3D motion compensated temperature maps.
Deleted: Motion compensated t

Deleted: overlayed

Comment [f4]: R2.2

Deleted: The transducer is situated on the right of the axial image. 

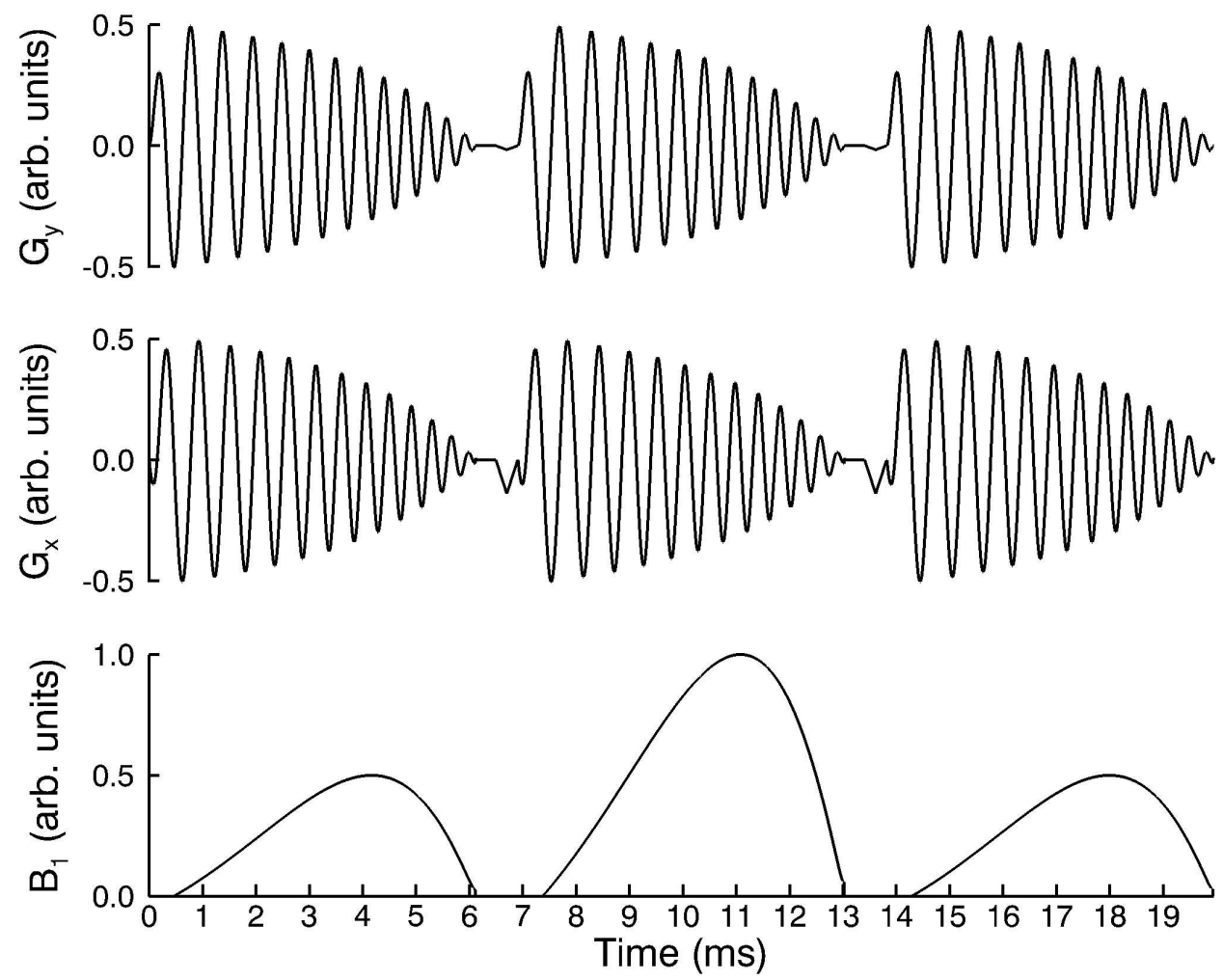

FIG. 1. Gradient and RF waveforms of the proposed 1-2-1 binomial 2D RF pulse train. The excitation k-space encoding is over 12 spiral turns, with a fat-water dephasing corresponding to $3 / 2 \pi$. The RF waveform is weighted according to the nonuniform sampling density. $90 \times 70 \mathrm{~mm}(600 \times 600 \mathrm{DPI})$ 
FIG. 2. Position of navigator (black rectangle) and imaging slice (white rectangle) for the (a) kidney and (b) liver in vivo slice tracking experiments. The navigator pencil beam was placed across the lower apex of the right kidney in the kidney experiment. For the liver experiment, the navigator was placed across the gallbladder and the surrounding fat at the bottom of the liver. The navigators intersected the axial imaging slice perpendicularly in both experiments. $121 \times 56 \mathrm{~mm}(450 \times 450 \mathrm{DPI})$ 
1

2

3

4

5

6

7

8

9

10

11

12

13

14

15

16

17

18

19

20

21

22

23

24

25

26

27

28

29

30

31

32

33

34

35

36

37

38

39

40

41

42

43

44

45

46

47

48

49

50

51

52

53

54

55

56

57

58

59

60

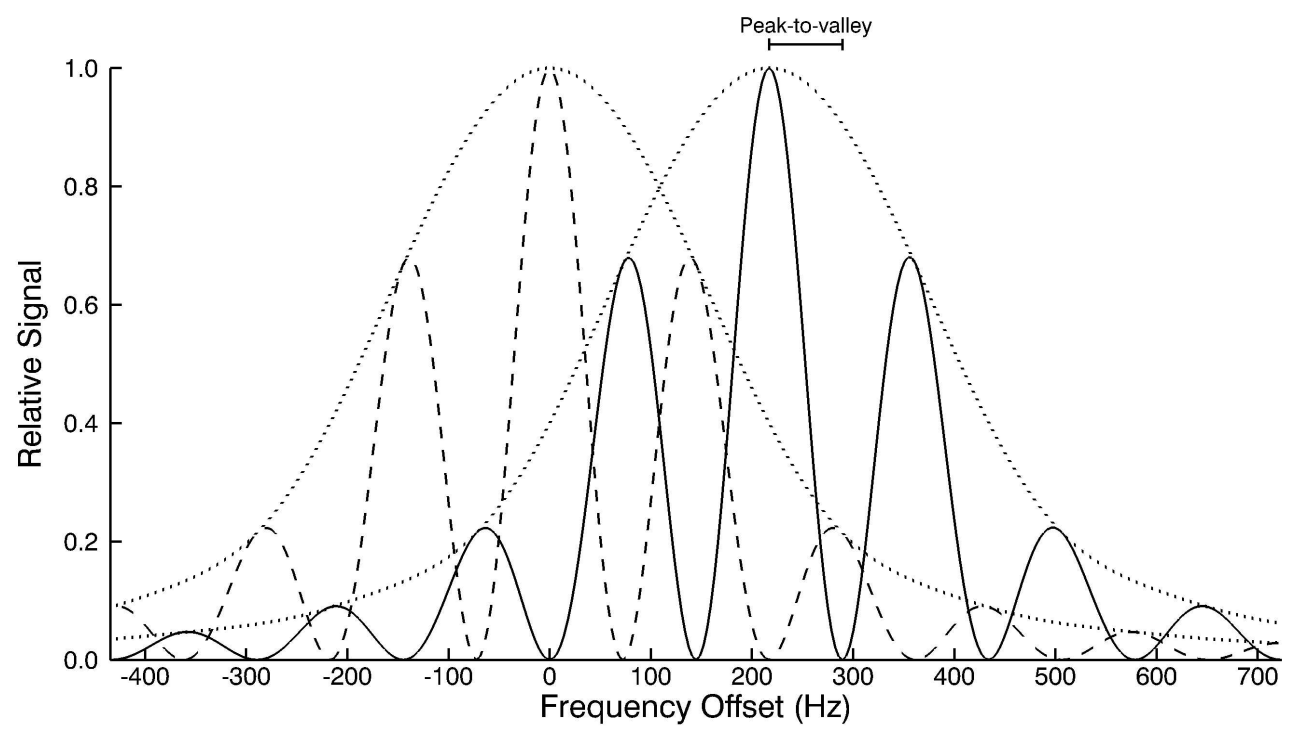

FIG. 3. Spectral response of the water-selective (dashed) and fat-selective (solid) pencil-beam navigator. The two envelopes (dotted) correspond to the non-selective navigator when excited at the fat and water resonance frequency. The distance between neighboring spectral peaks and valleys of $72 \mathrm{~Hz}$ is also shown. $114 \times 63 \mathrm{~mm}(600 \times 600 \mathrm{DPI})$ 
FIG. 4. Excitation profile of the spectrally selective pencil-beam excitation at frequency offsets of 0 $\mathrm{Hz}$ (solid), $145 \mathrm{~Hz}$ (dotted) and $290 \mathrm{~Hz}$ (dashed). The excitation profiles of both the conventional non-selective and the spectral-selective pencil-beam excitation coincide at the frequency offsets corresponding to the spectral peaks of Fig. 3. The full width at half maximum (FWHM) of the excitation profiles at 0 and $145 \mathrm{~Hz}$ are also indicated. $114 \times 63 \mathrm{~mm}(600 \times 600 \mathrm{DPI})$ 
39

40
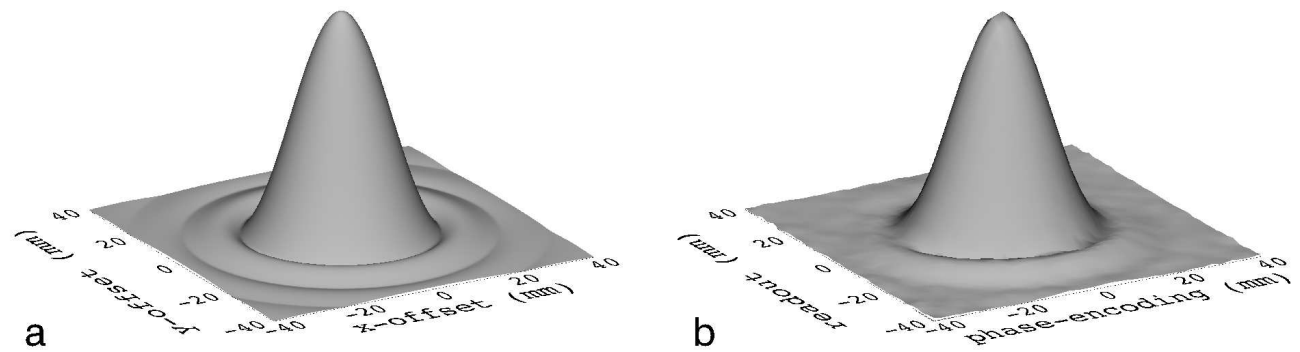

FIG. 5. Comparison of the (a) theoretical and (b) experimental central peak of the on-resonance excitation profile as viewed perpendicularly to the pencil-beam direction. The excitation profile shows a good spatial localization with the central peak in the expected form of a jinc function. $161 \times 43 \mathrm{~mm}(600 \times 600 \mathrm{DPI})$ 

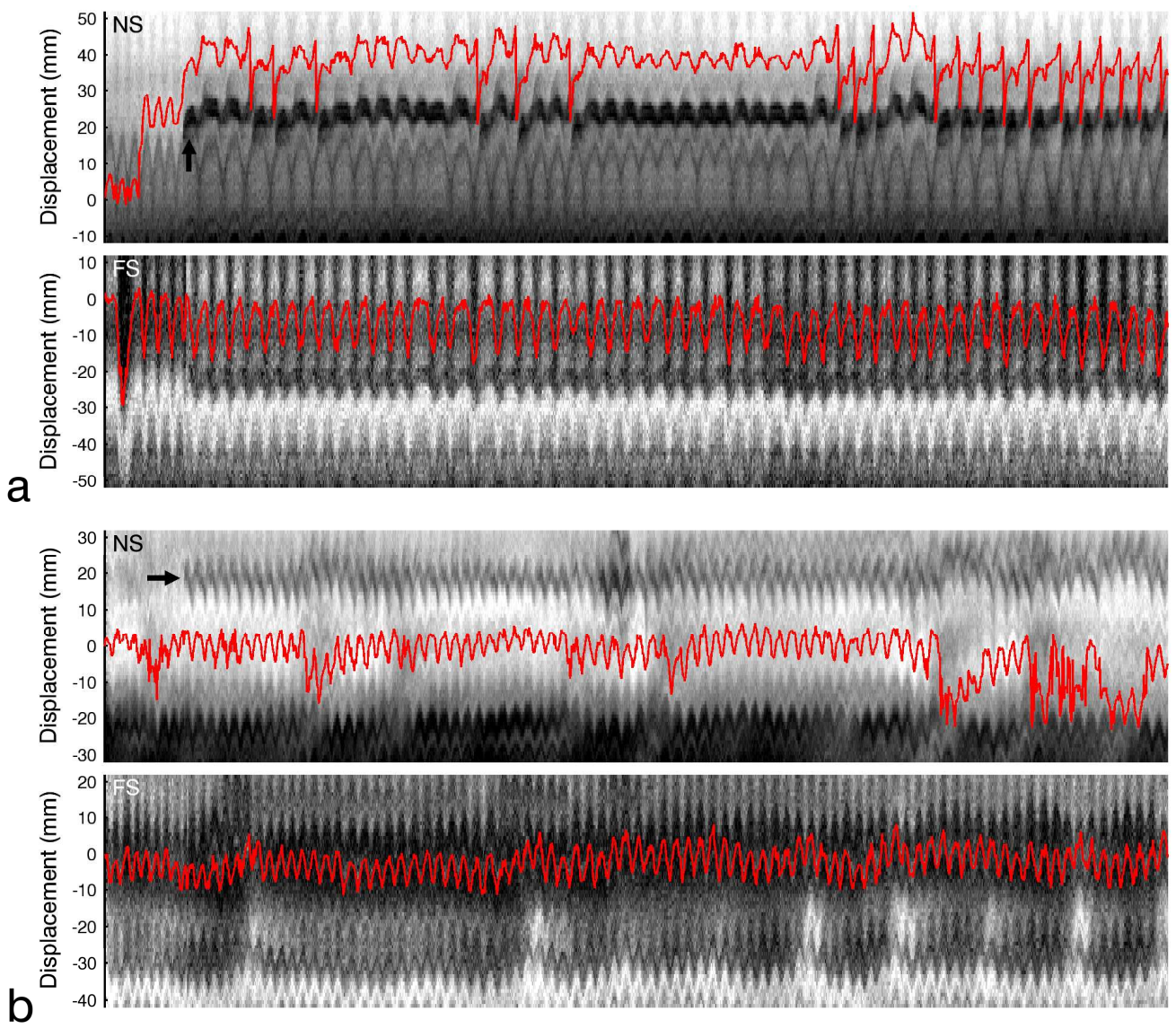

FIG. 6. Comparison of the slice tracking performance of the conventional spectrally non-selective navigator (NS) and the fat-selective navigator (FS) when placed directly on (a) the kidney and (b) the liver. The navigator profiles are plotted vertically with subsequently acquired profiles stacked after each other and the dynamic scan number increasing towards the right. The displacements estimated based on the correlation of the navigator profiles are depicted in red. The navigator profile obtained by the conventional non-selective navigator is altered significantly by the onset of the water-selective MR imaging (indicated by arrow). This effect is much less pronounced for the fat-selective navigator profile. Note that the displacement estimates were shifted relative to the navigator profiles in order not to interfere with the essential features of the navigator profiles. $163 \times 142 \mathrm{~mm}(600 \times 600 \mathrm{DPI})$ 


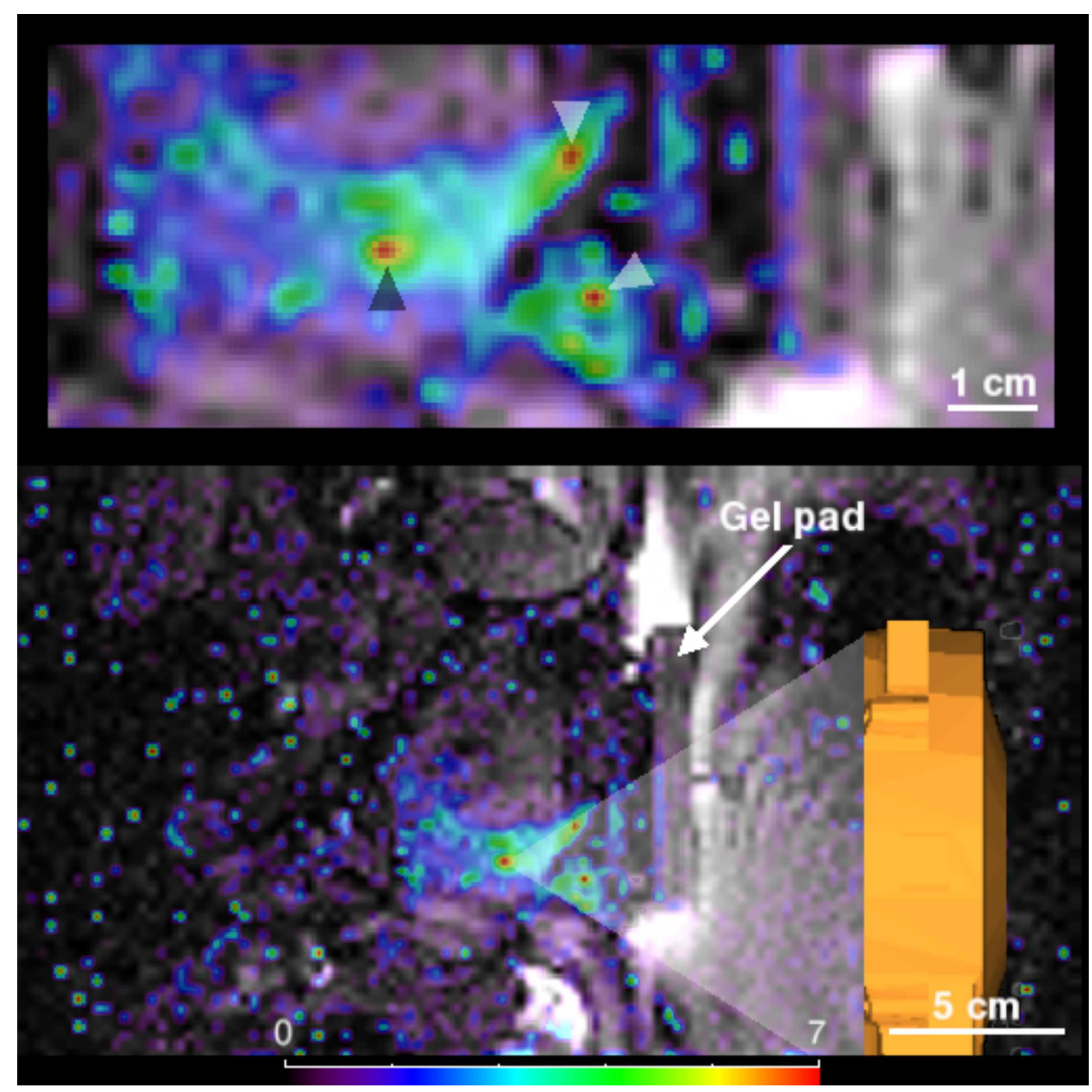

FIG. 7. Temperature map of the kidney at the end of a beam-steered HIFU sonication. The motion compensated temperature map is overlaid in color on the axial magnitude image. The transducer and the semi-transparent beam-path overlay as well as the location of the gel pad are shown to clarify the experimental setup. A close-up of the temperature map within the beam path is also shown (top) with the black arrowhead indicating the position of the focus within the kidney. Two additional hotspots (white arrowheads) due near-field heating can also be seen. The proposed fatselective navigator was used to provide the necessary through-plane motion estimate. $86 \times 86 \mathrm{~mm}(600 \times 600 \mathrm{DPI})$ 
FIG. 8. Temperature rise in the target point within the porcine kidney during HIFU sonication with (top) and without (middle) beam steering. The kidney displacement in the SI direction obtained from the fat-selective navigator data is also shown (bottom). The temperature in the target point of the kidney was obtained from 3D motion compensated temperature maps. $76 \times 114 \mathrm{~mm}(600 \times 600$ DPI $)$ 